\title{
Quantification of multiphoton and fluorescence images of reproductive tissues from a mouse ovarian cancer model shows promise for early disease detection
}

\author{
Travis W. Sawyer \\ Jennifer W. Koevary \\ Faith P. S. Rice \\ Caitlin C. Howard \\ Olivia J. Austin \\ Denise C. Connolly \\ Kathy Q. Cai \\ Jennifer K. Barton
}




\title{
Quantification of multiphoton and fluorescence images of reproductive tissues from a mouse ovarian cancer model shows promise for early disease detection
}

\author{
Travis W. Sawyer, ${ }^{a}$ Jennifer W. Koevary, ${ }^{b}$ Faith P. S. Rice, ${ }^{b}$ Caitlin C. Howard, ${ }^{b}$ Olivia J. Austin, ${ }^{b}$ \\ Denise C. Connolly, ${ }^{c}$ Kathy Q. Cai, ${ }^{c}$ and Jennifer K. Barton ${ }^{\mathrm{a}, \mathrm{b}, *}$ \\ aUniversity of Arizona, College of Optical Sciences, Tucson, Arizona, United States \\ bUniversity of Arizona, Department of Biomedical Engineering, Tucson, Arizona, United States \\ ${ }^{c}$ Fox Chase Cancer Center, Philadelphia, Pennsylvania, United States
}

\begin{abstract}
Ovarian cancer is the deadliest gynecologic cancer due predominantly to late diagnosis. Early detection of ovarian cancer can increase 5-year survival rates from $40 \%$ up to $92 \%$, yet no reliable early detection techniques exist. Multiphoton microscopy (MPM) is a relatively new imaging technique sensitive to endogenous fluorophores, which has tremendous potential for clinical diagnosis, though it is limited in its application to the ovaries. Wide-field fluorescence imaging (WFI) has been proposed as a complementary technique to MPM, as it offers high-resolution imagery of the entire organ and can be tailored to target specific biomarkers that are not captured by MPM imaging. We applied texture analysis to MPM images of a mouse model of ovarian cancer. We also conducted WFI targeting the folate receptor and matrix metalloproteinases. We find that texture analysis of MPM images of the ovary can differentiate between genotypes, which is a proxy for disease, with high statistical significance $(p<0.001)$. The wide-field fluorescence signal also changes significantly between genotypes $(p<0.01)$. We use the features to classify multiple tissue groups to over $80 \%$ accuracy. These results suggest that MPM and WFI are promising techniques for the early detection of ovarian cancer. $\odot$ The Authors. Published by SPIE under a Creative Commons Attribution 4.0 Unported License. Distribution or reproduction of this work in whole or in part requires full attribution of the original publication, including its DOI. [DOI: 10.1117/1.JBO.24.9.096010]
\end{abstract}

Keywords: multiphoton imaging; fluorescence imaging; ovarian cancer; mouse model.

Paper 190174R received Jun. 1, 2019; accepted for publication Sep. 13, 2019; published online Sep. 30, 2019.

\section{Introduction}

\subsection{Burden of Ovarian Cancer}

Despite concerted efforts to improve patient outcomes, ovarian cancer remains the deadliest gynecologic malignancy in the United States, with a five-year survival of $<50 \% .^{1,2}$ Ovarian cancer can become advanced before causing signs or symptoms, and a large majority of patients have already experienced spread of their disease to local or distant tissues at initial diagnosis, resulting in a significantly poorer prognosis. ${ }^{3}$

Therefore, there is strong interest in ovarian cancer screening, with the ultimate goal of identifying early stage tumors, while the patient is still asymptomatic, allowing more effective treatment. Various screening modalities have been investigated to reduce the burden of the disease including physical examination, transvaginal ultrasound, and serum tumor marker measurement (most commonly CA-125). ${ }^{4,5}$ Other screening tests and multimodal protocols have also been investigated; however, at this time, no routine screening is recommended in average-risk patients. ${ }^{6}$ As such, there remains a strong need for a high-quality, minimally invasive modality for effective detection of early-stage ovarian malignancies.

*Address all correspondence to Jennifer K. Barton, E-mail: barton@email .arizona.edu

\subsection{Multiphoton Imaging}

Several different optical imaging techniques have been used to probe ovarian tissue health including fluorescence, ${ }^{7}$ multispectral imaging, ${ }^{8}$ confocal imaging, ${ }^{9}$ photoacoustic imaging, ${ }^{10}$ and optical coherence tomography. ${ }^{11}$ One technique that has shown promise in the identification of abnormal ovarian tissue is multiphoton microscopy (MPM). ${ }^{12,13}$ MPM is based on the molecules in tissue interacting with more than one photon simultaneously, allowing for signal generation at a higher energy than the illuminating light. One type of MPM is second-harmonic generation (SHG) in which contrast is formed by the nonlinear scattering of noncentrosymmetric structures. ${ }^{14}$ The nonlinear nature of the scattering in SHG results in emission light with precisely twice the frequency of the excitation light.

Another form of MPM is two-photon excited fluorescence (2PEF) in which an endogenous fluorophore absorbs two photons nearly simultaneously to generate fluorescence emission at a higher frequency than the incident light. Due to vibrational relaxations and other losses, the frequency of the fluorescent light undergoes a Stokes shift to slightly less than twice the illumination frequency. 2PEF can be generated by endogenous fluorophores, eliminating the possibility of toxicity or other side effects from exogenous contrast agents and simplifying the imaging procedure. Endogenous fluorophores in the body include proteins containing aromatic amino acids (tryptophan, tyrosine, and phenylalanine), metabolic co-factors such as nicotinamide adenine dinucleotide and flavin adenine dinucleotide, 
structural proteins such as collagen and elastin, and a variety of other molecules including vitamins and lipopigments. ${ }^{15,16}$

For both SHG and 2PEF, three-dimensional images are formed by scanning the focused, short-pulsed excitation beam across the tissue. The probability of two-photon interaction scales with the square of the irradiance. ${ }^{17}$ Due to the irradiance of the beam sharply decreasing with distance from the beam focus, this effect allows for probing a small volume of the sample, without exciting regions of the sample outside the focal plane. ${ }^{18}$ The necessity of high numerical aperture to generate efficient two-photon processes (probability of excitation) yields lateral resolutions on the order of hundreds of nanometers. ${ }^{19,20}$ $2 \mathrm{PEF}$ and SHG have the advantage of high resolution, depthresolved imaging, with an imaging depth greater than confocal imaging. The increased depth of imaging is achieved using near-infrared light, which penetrates deeper than the ultraviolet or visible wavelengths typically used in single-photon techniques such as confocal microscopy and surface fluorescence imaging. Additionally, at near-infrared wavelengths, there is minimal absorption and reduced tissue damage.

\subsection{Wide-field Fluorescence Imaging}

MPM imaging has a limited field of view, posing challenges for application as a screening tool. One solution is to couple MPM with a wide field-of-view modality, such as wide-field fluorescence imaging (WFI). WFI illuminates a large area of tissue, for rapid imaging with little or no scanning. In the case of a mouse, we are able to view the entire reproductive tract including uterus, oviducts, and ovaries in a single image. The addition of targeted fluorescent dyes can provide both qualitative and quantitative information on desired markers in the tissue and overall tissue composition.

A number of different light sources and filters can be used to separate the excitation and emission of fluorophores related to ligands of interest. In the scope of ovarian cancer, the folate receptor is an interesting target, as folate binding protein is overexpressed in advanced ovarian cancers. ${ }^{21}$ Knowing this trend in late disease suggests that the protein may be altered with early disease as well. In addition, matrix metalloproteinases (MMPs) play a pivotal role in tumor growth and the multistep processes of invasion and metastasis in general cancers. ${ }^{22}$

\subsection{Image Analysis}

Several different approaches have been applied for quantitative analysis of MPM images. One of the most direct is to threshold the images based on brightness to evaluate fibrosis of the collagen network. ${ }^{23,24}$ Another common technique is to employ frequency analysis, which quantifies the global spatial frequency content of an image. ${ }^{25-27}$ Recently, techniques focusing on local image features have been applied for tissue health classification. These include wavelet ${ }^{28,29}$ and curvelet analysis ${ }^{30}$ and convolving the images with a set of filter patches at different scales and orientations. $^{31}$

MPM generates contrast that shows changes in endogenous cellular fluorescence (2PEF) and collagen structure (SHG) as a result of ovarian cancer. ${ }^{32-34}$ For example, SHG has been used to show that normal ovaries have thin collagen fibers organized in a net-like structure, whereas malignant ovaries have a denser, wavy collagen structure, which may result from recruitment of activated fibroblasts to the outer rim of the tumor. ${ }^{26,32,34,35}$
Therefore, analysis of MPM images is focused on quantitatively assessing these structural differences.

One of the most promising approaches to quantitation of MPM images of ovarian tissue is image texture analysis. Texture analysis in image processing is a general method for describing the local variations in image brightness. Texture characterizes the spatial distribution of the tones in an image. ${ }^{36}$ A number of techniques for texture analysis of images have been developed; these can be generally categorized into three groups: statistical, spectral, and structural methods. Statistical methods are based on analyzing image histograms by computing their statistical moments and other properties. ${ }^{37}$ These approaches are best suited to characterize features such as inhomogeneity and contrast. Spectral methods apply autocorrelation and Fourier analysis to evaluate periodic features of an image. Finally, structural approaches decompose the image into a set of subpatterns, arranged according to certain placement rules.

Here we use MPM and WFI to image a mouse model of ovarian cancer in vivo. WFI and MPM images are collected for both the ovaries and the oviducts and features are extracted from MPM using texture analysis and from WFI by calculating the magnitude of the fluorescence emission. We find that these features can differentiate between mouse groups with high statistical significance. These features are then used to build a tissue classifier. The results suggest that texture analysis of MPM images and intensity of target fluorescence images may be useful as an aid for ovarian cancer screening.

\section{Methods}

\subsection{Mouse Model}

For this experiment, we used a transgenic mouse model in which females spontaneously develop bilateral epithelial ovarian cancer using the TgMISIIR-TAg (TAg) transgene. ${ }^{38,39}$ This mouse model has been shown to exhibit both genotypic and phenotypic similarities with human ovarian carcinoma. ${ }^{40}$ Male TAg mice were bred to female C57Bl/6 (wild type) mice. This resulted in production of offspring that either harbor the TAg transgene (TAg+) or are the wild type. Female offspring of both genotypes were injected with the vehicle (sesame oil) or 4-vinylcyclohexene diepoxide (VCD) dissolved in sesame oil at a concentration of $80 \mathrm{mg} / \mathrm{kg}$ for 20 days beginning at postnatal day 7. VCD was used to destroy preantral follicles, resulting in early ovarian failure. VCD has previously been used as a model for menopause. ${ }^{41}$ The same cohort of mice was imaged at 4 and 8 weeks. For brevity, we refer to different groups in the figures by abbreviating (age-genotype-treatment). For example, 4WV refers to 4 weeks of age, wild type treated with VCD and 8TS refers to 8 weeks of age, TAg+ treated with sesame oil.

With this procedure, we have eight different groups to compare $(2 \times 2 \times 2$ for age, genotype, and treatment). This poses an interesting challenge for class separation based on image analysis, as we expect the structure of the ovary and oviduct to change due to each of these three processes. A total of 53 mice were imaged at both time points, yielding 13 samples per group except for the wild type sesame-oil-treated mice, of which there were 14 (Table 1 ).

\subsection{Surgical Procedure}

All procedures were performed according to an IACUCapproved protocol. Prior to surgery, ketamine $(60 \mathrm{mg} / \mathrm{kg}) /$ 
Table 1 Number of mice for each experimental group imaged, total mice are shown in brackets, if different. Some images were excluded due to saturation, as described in Sec. 2.6.

\begin{tabular}{lccccc} 
& \multicolumn{2}{c}{ VCD } & & \multicolumn{2}{c}{ Sesame oil } \\
\cline { 2 - 3 } \cline { 6 - 6 } Age & TAg+ & Wild type & & TAg+ & Wild type \\
\hline Week & 13 & 13 & & $12(13)$ & 14 \\
Week & $11(13)$ & 13 & 13 & 14 \\
\hline
\end{tabular}

xylazine $(7.5 \mathrm{mg} / \mathrm{kg})$ was administered to mice through intraperitoneal injection. The right dorsal side of the body was shaved with clippers from the ribs to the hind limb and then treated with Nair hair remover for less than one minute. Nair was removed using water and cotton pads. Surgical scrub was performed with three alternating scrubs of chlorhexidine and alcohol with the final being a spray or wipe of chlorhexidine.

Mice were then placed on a custom-built heating pad where they were given isoflurane, USP (Piramal, Mumbai, India) anesthetic through a nose cone and draped with a sterile surgical drape. Isoflurane was initially set at $1 \%$ to $3 \%$ volume (maintain oxygen at 0.6 to $0.8 \mathrm{~L} / \mathrm{min}$ ) to ensure surgical level of anesthesia prior to skin incision. Thereafter isoflurane level was lowered to $1.5 \%$ volume $(98.5 \%$ oxygen) for surgical maintenance. Anesthesia levels were monitored by observing the animals' respiration rate and pattern during surgery and imaging.

An 8-mm incision was made in skin followed by blunt dissection under the skin. Then a second incision was made in the peritoneum. Directly under the incision, in the peritoneal cavity, is an adipose pad attached to the ovary. A suture was placed through the fat pad and the tissue gently placed so that the ovary was facing upward (fat downward) on a sterile spoon and the suture was secured in place using Tegaderm film (3M, Maplewood, Minnesota). The opposite end of the spoon was secured to stabilize the ovary and isolate the ovary from body motion for imaging. Great care was taken to be gentle with the adipose tissues to avoid damage to blood vessels, ovaries, and surrounding tissue. Fluorescent dyes were applied, incubated, and rinsed as described below. Then a small amount of sterile Surgilube (HR Pharmaceuticals, York, Pennsylvania) was placed on the ovary and the ovary covered with a sterile coverslip for imaging. Following imaging, the ovary was rinsed with sterile saline, the suture clipped, and the ovary returned to the anatomical position. Antiadhesion Seprafilm (Genxyme, Cambridge, Massachusetts) was placed in the incision. The peritoneum was closed with absorbable catgut suture and the skin closed with staples. Topical antibiotic was administered to the wound. The surgical externalization and stabilization process has been described in more detail previously. ${ }^{42}$

\subsection{Dye Application and Wide-Field Fluorescence Imaging}

A number of commercial fluorescent dyes are available for tissue characterization. We are most interested in tissue markers that are potentially up-regulated in cancer so that we can understand the expression of these markers in the mouse models that we use. The tissue markers that were of highest interest to us and had commercially available dyes including folic acid (FolateRSense; Perkin Elmer, Waltham, Massachusetts), ${ }^{43}$ and MMPs (MMPSense 680; Perkin Elmer). ${ }^{44}$ A preliminary study prior to the work published here was conducted to confirm that these dyes produced a detectable signal using the imaging system described below.

Dyes were prepared according to package instructions and kept in foil wrapped tubes to protect from ambient light. Ovaries were surgically exposed in live animals and a sterile pipette tip was used to place $50 \mu \mathrm{L}$ of dye onto each ovary, or $100 \mu \mathrm{L}$ of dye into the body cavity (for control organs), resulting in a total of $100 \mu \mathrm{L}$ of dye per animals. Organs were allowed to incubate with dye for $10 \mathrm{~min}$ in darkness.

WFI was then performed with an MVX10 microscope with a DP80 digital camera (Olympus, Tokyo, Japan) and ImageX software. Images were taken at exposure times of $2 \mathrm{~s}$ (for MMPSense dye) or $0.1 \mathrm{~s}$ (for FolateRSense). Magnification was set to 0.8. Each channel was set to un-gated and a frequency of 100,000. Light was filtered using the microscope's CY5.5 filter set, featuring a cut-on wavelength of $685 \mathrm{~nm}$, excitation band of 635 to $675 \mathrm{~nm}$, and emission band of 696 to $736 \mathrm{~nm}$. The excitation spectrum of these dyes does not overlap with the wavelength used for MPM, as described next; thus, there is no cross interaction between fluorescence and MPM studies, as confirmed by examining both stained and unstained tissues with MPM.

\subsection{Multiphoton Imaging}

MPM imaging was performed with a single-beam multiphoton microscope (TrimScope, LaVision BioTec, Bielefeld, Germany) using a titanium:sapphire laser light source (Chameleon Ultra2, Coherent, UK) that was coupled to the scanner unit, with a pulse width of 120 femtoseconds at the sample. The laser intensity was adjusted to $35 \mathrm{~mW}$ average power with an electro-optical modulator (EOM 350-80; Conoptics, Danbury, Connecticut). A water-immersion, coverslip-corrected, 20× magnification, 0.95-NA objective (MRD77200 Nikon, Tokyo JP) was used for imaging. The excitation wavelength was set to $780 \mathrm{~nm}$, and SHG and 2PEF image data were recorded simultaneously. A bandpass filter (FF01-377/50; Semrock, Rochester, New York) and a dichroic mirror (Di01-R405-25X36; Chroma, Bellows Falls, Vermont) were used to collect light for SHG, and a bandpass filter (HQ450/100M-2p-25; Chroma) and a dichroic mirror (505dcxr; Chroma) were used to collect light for 2PEF. Images were taken at $5-\mu \mathrm{m}$ depth increments from the surface of the tissue to 50 - to $100-\mu \mathrm{m}$ depth. Imaging was completed in less than 5 min per image stack. Images were collected at the center of the ovary, at the proximal side of the ovary near the oviduct, and at the oviduct. All images had a $400 \mu \mathrm{m} \times 400 \mu \mathrm{m}$ field of view and contained $1024 \times 1024$ pixels with 14-bit gray scale resolution.

Note that with the limited field of view of MPM, it cannot be determined whether the specific subregion of the organ captured by the image area contains active tumor tissue. Histology indicated that mice with disease ranged from $2 \%$ to $50 \%$ tumor coverage (see Sec. 5) Thus we cannot claim the comparisons between genotypes are directly translatable to comparisons between malignant and nonmalignant tissue. Although early changes may still be observed in the entire organ, we continue to refer to the genotypes as the two experimental groups for correctness. 


\subsection{Histology}

Following imaging at 8 weeks of age, mice were euthanized via $\mathrm{CO}_{2}$ and the entire reproductive tract was removed. Because the mice survived beyond their first imaging session, no histology is available for comparison with 4 week images. Histology from a separate cohort of mice euthanized at 4 weeks $(n=47$, at least 10 in each combination of genotype and treatment) was instead utilized for comparison. The organs were fixed in neutral-buffered formalin and paraffin embedded and sectioned for analysis. Immunohistochemical (IHC) staining of mouse reproductive tract tissue sections was performed with antibodies recognizing SV40 TAg, PAX8, and $\alpha$-inhibin. Tissues were stained with the above antibodies using established protocols for immunoperoxidase-based detection of antibody binding [described previously in Connolly 2003 (TAg and $\alpha$-inh) ${ }^{38}$ and Gabbasov 2018 (PAX8) ${ }^{45}$ ].

Algorithms were developed to quantify TAg or PAX8 staining across all mice. The IHC stained sections were scanned and images acquired using the Vectra Multispectral Imaging system (Version 2.0.8, Perkin Elmer, Waltham, Massachusetts). For quantification of IHC staining, images were captured with a $40 \times$ objective and analyzed using InForm software (Version 2.1.1, Perkin Elmer, Waltham, Massachusetts). Algorithms to segment the tissue compartments (ovary, nonovary, oviduct, nonoviduct, and nontissue) and for subcellular compartment separation were created by machine learning using InForm Software. For tissue segmentation training, a small batch of 20 test images were manually identified as ovary, oviduct, or other tissue, as well as nontissue elements including glass histological artifacts. The cells in ovary or oviduct segments were scored to quantify IHC staining for TAg and PAX8 in the nuclei only. The Inform software scores these cells according to DAB intensity ( 0 to 3 ) and extent $(0 \%$ to $100 \%)$ to compute a composite $H$-score. When the algorithm template of segmentation and scoring for the test cases was sufficiently accurate $(>95 \%)$, it was run on all images and compared with manual scoring as a confirmation. Then entire image $\mathrm{H}$-scores were exported to excel for analysis. Prism statistical analysis software (Graphpad, San Diego, California) was then used to determine age- and treatment-specific differences in staining across all groups.

The H\&E, Tag, and $\alpha$-inhibin stained reproductive tract tissue sections were reviewed and evaluated for the presence and extent (percent) of tumor based on cell morphology and the presence of TAg protein. The $\alpha$-inhibin staining was evaluated to confirm the absence of $\alpha$-inhibin protein in tumor tissue and its presence in residual normal follicles. The ovaries of 4- and 8-week-old wild type and MISIIR-TAg transgenic mice, treated with the vehicle (soybean oil) or VCD were evaluated to assess the number of follicles, corpora lutea, and atretic follicles in each case.

\subsection{Image Processing}

\subsubsection{Wide-field fluorescence imaging}

Images were examined by eye and excluded from analysis if saturation occurred. Analysis was performed using ImageJ software. ${ }^{46}$ For each organ, a $40 \times 40$ pixel square was placed in the center of the organ and the mean signal intensity in the region was recorded. Each pixel was $6.45 \mu \mathrm{m}$ in width, so the analyzed area was $258 \mu \mathrm{m} \times 258 \mu \mathrm{m}$.

\subsubsection{Texture analysis of multiphoton images}

We selected MPM images for analysis by selecting the image with the highest average signal throughout the image stack in the depth $(z)$ direction, which did not show signs of saturation. Three image stacks saturated throughout the majority of the depth $(80 \%)$ were excluded from analysis, as indicated in Table 1. Analysis was performed by an observer who was blinded to the classification of the mice.

We applied two methods of texture analysis to extract features from the acquired MPM images. The first is based on constructing and analyzing the gray-level co-occurrence matrix (GLCM). ${ }^{36}$ The GLCM is a spatial histogram that describes the distribution of gray-level values in an image. Each entry in the $\operatorname{GLCM} p(i, j \mid d, \theta)$ corresponds to the probability of a pixel with a gray level of $(i)$ being a distance $(d)$ pixels away from a neighboring pixel with a gray level of $(j)$ in the $(\theta)$ direction. With an image quantized into $N_{g}$ gray levels, the GLCM is an $N_{g} \times N_{g}$ matrix. For a two-dimensional (2-D) image, four directions for $(\theta)$ are possible: $0 \mathrm{deg}, 45 \mathrm{deg}, 90 \mathrm{deg}$, and $135 \mathrm{deg}$. In this study, we fixed $(d)$ at one pixel (3.9 $\mu \mathrm{m}$ in object space) and computed the GLCM for the four possible directions. All images were normalized and quantized to 8-bit (intensity ranging between 0 and 255). From the GLCM, we then computed 13 texture features introduced by Haralick et al., ${ }^{36}$ averaged over the four directions for $\theta$.

We computed a second set of features by analyzing the discrete fast Fourier transform (FFT) of the image in 2-D, which describes the distribution of spatial frequencies present in an image. After applying the FFT, the image was normalized so that all pixel values summed to one. Then we summed the pixel values in a small disk centered at the origin and recorded the result. The radius of this disk was iteratively increased, and the summed pixel value within its area was recorded until the disk radius had reached $80 \%$ of the image half-width, beyond which primarily noise remains. This was effectively the cumulative distribution function (CDF) of the energy density as a function of radial spatial frequency; taking the difference between the values of this curve for any two radial frequencies gives the proportion of energy contained within a specific frequency band. Images that were highly homogenous had higher energy density associated with lower spatial frequencies. On the other hand, images with more inhomogeneity had more energy density corresponding to higher spatial frequency. We then parameterized the distribution by fitting the CDF curve to the following equation, which we qualitatively found to fit the curve well:

$y=\alpha x^{\beta}+\gamma$,

where $y$ is the value of the CDF for a given spatial frequency $x$. The frequency distribution was thus described by the three features: $\alpha, \beta$, and $\gamma$, which were used to differentiate between the different experimental groups. Combining these with the 13 Haralick features gave a set of 16 total texture features. The analyses were completed in Python using a computer with an Intel Core I-4710HQ CPU $(2.50 \mathrm{GHz})$ and $16 \mathrm{~GB}$ DDR3L memory.

\subsection{Feature Selection and Classification}

Once feature vectors were created, redundant features were removed by calculating the correlation matrix for the feature set. For each pair of features that were highly correlated 
(correlation $>0.85),{ }^{47}$ one feature from the set was removed (the feature which yielded a lower average $p$-value using the statistical test described above). Note that the WFI image data were not used in the classification process, as these would be more appropriate to flagging suspicious areas, which then could be further probed with MPM.

We exhaustively tested the classification performance of feature subsets consisting of six or fewer features, as the literature suggests that high performance can typically be achieved with two to five features. ${ }^{47-49}$ To evaluate how well a set of features could separate different classes, we used the trace of the ratio of the between-class scatter $\left(S_{B}\right)$ and within-class scatter $\left(S_{w}\right)$, which has successfully been applied in similar scenarios. ${ }^{11,47}$

To classify the data, we used linear discriminant analysis (LDA), ${ }^{50}$ which has been applied frequently in the scope of medical image classification. ${ }^{11,51,52}$ For our classification, we reduced the dimension to the linear discriminants that account for $99 \%$ of the variance in each case, before generating the optimal decision boundary. To validate the model, we used leave-one-out cross validation. Additional details regarding our classification process have been previously described. ${ }^{11}$

\subsection{Statistics}

Statistical analysis for the texture analysis for MPM and WFI images was completed using one-way analysis of variance (ANOVA) to test differences between individual groups, as well as using a linear mixed effects (LME) model with random intercepts to account for within-subject and three-way interactions between age, genotype, and treatment. The latter is used to attempt to offset the confounding effects of the multiple experimental groups, e.g., heteroscedasticity, or unequal variance among groups. It is also a tool to assess an overall influence of age, treatment, or genotype that may not be apparent between two individual groups. The Python statsmodel library (Python Software Foundation, Delaware) was used to construct the LME model. The resulting model produced postestimation inference via Wald tests and confidence intervals on the variables, profile likelihood analysis, likelihood ratio testing, and the Akaike information criterion to assess the quality of the model. Results from LME models that did not converge were discarded. Note that the Wald test statistic assesses the Fisher information of a variable at the maximum-likelihood estimation point. Essentially, the Wald test evaluates whether an explanatory variable is significant in the construction of the model. ${ }^{53}$ This is fundamentally different than a $t$-test, which tests the likelihood of whether two means are different.

An argument could be made for conducting a paired statistical comparison between age groups, as the same mice were imaged at both time points. However, it is not guaranteed that the same region of the organ was imaged at both time points. Therefore, it is possible that inhomogeneity in the tissue could result in variations in the texture features. Thus we conducted unpaired statistical tests. Histology results were analyzed by one-way ANOVA and the nonparametric Kruskal-Wallis test followed by Dunns posttest. Differences were considered statistically significant for $p<0.05$ (denoted $*), p<0.01$ (denoted $* *$ ), $p<0.001$ (denoted $* * *$ ), and $p<0.0001$ (denoted $* * * *$ ).

\section{Results and Discussion}

\subsection{Histopathology Analysis}

H\&E stained sections of an ovary are shown for a wild type [Fig. 1(a)] and a TAg mouse [Fig. 1(b)]. Higher power magnification images are shown for the same wild type [Fig. 1(c)] and

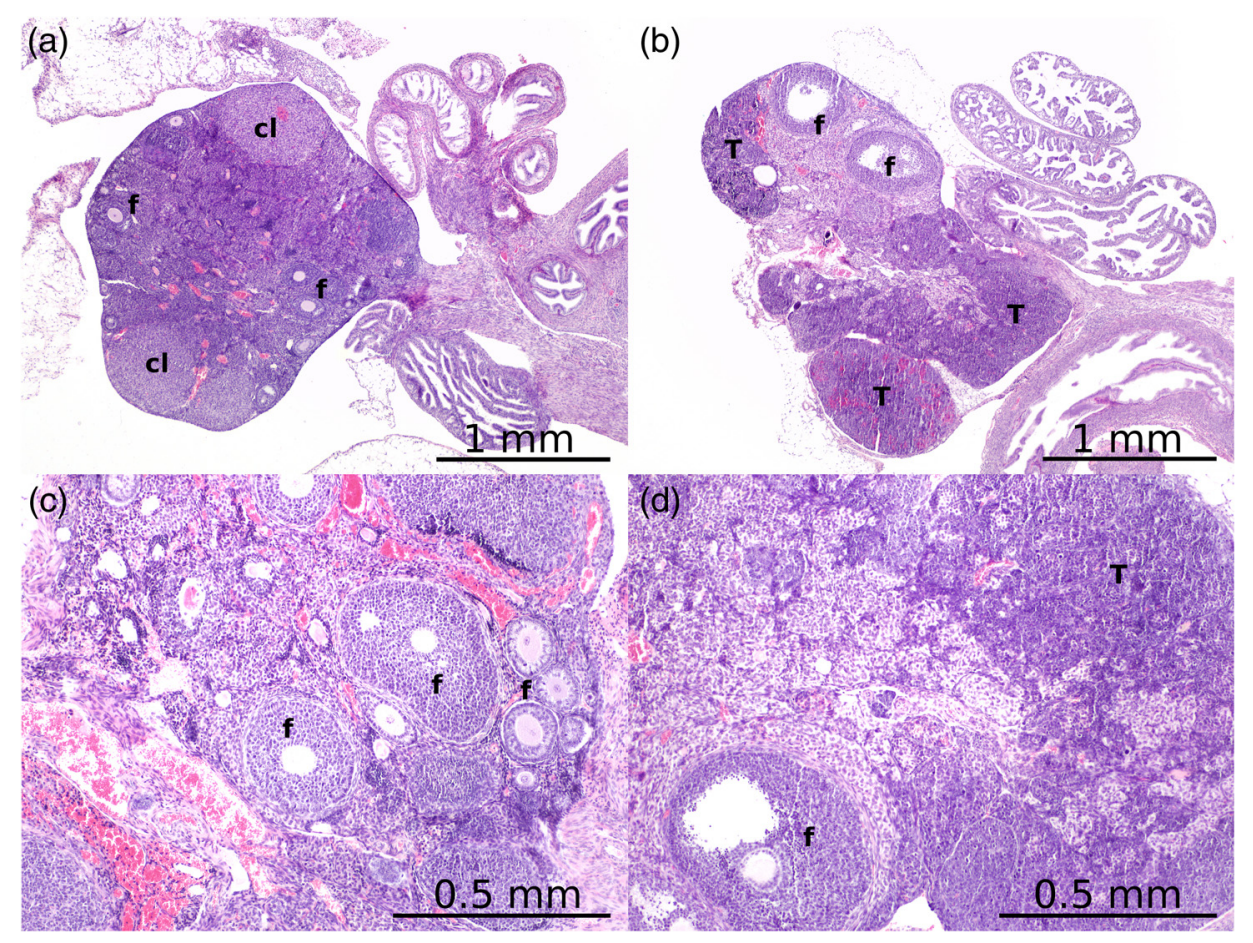

Fig. 1 H\&E staining of an ovary at $4 \times$ for (a) WT and (b) TAg mouse, and at a magnification of $10 \times$ for the same (c) wild type and (d) TAg mouse. Follicles (f), corpora lutea (cl), and tumor (T) are labeled as indicated. 
TAg [Fig. 1(d)] tissues. The wild type ovary has normal appearance, with well-structured follicles and corpora lutea [Figs. 1(a) and 1(c)]. In contrast, the TAg ovary has obvious infiltration of neoplastic cells [Figs. 1(b) and 1(d)] with hyperchromatic nuclei and eosinophilic cytoplasm. Overall, much of the normal structure of the organ has been replaced by neoplastic cells. IHC staining for TAg shows the presence of the TAg protein (Fig. 2), confirming the expression of the TAg transgene in the mice with tumors. Staining for PAX8 also confirms the onset of disease in TAg mice (Fig. 3). Results from the segmentation and scoring process are shown in Fig. 4 for a TAg and wild type mouse at 8 weeks of age, treated with sesame oil.

Summary statistics of the $H$-score for TAg and PAX8 staining for wild type and TAg transgenic mice dosed with both the vehicle (sesame oil) and VCD are shown in Fig. 5. We see significant differences primarily between genotype and age and
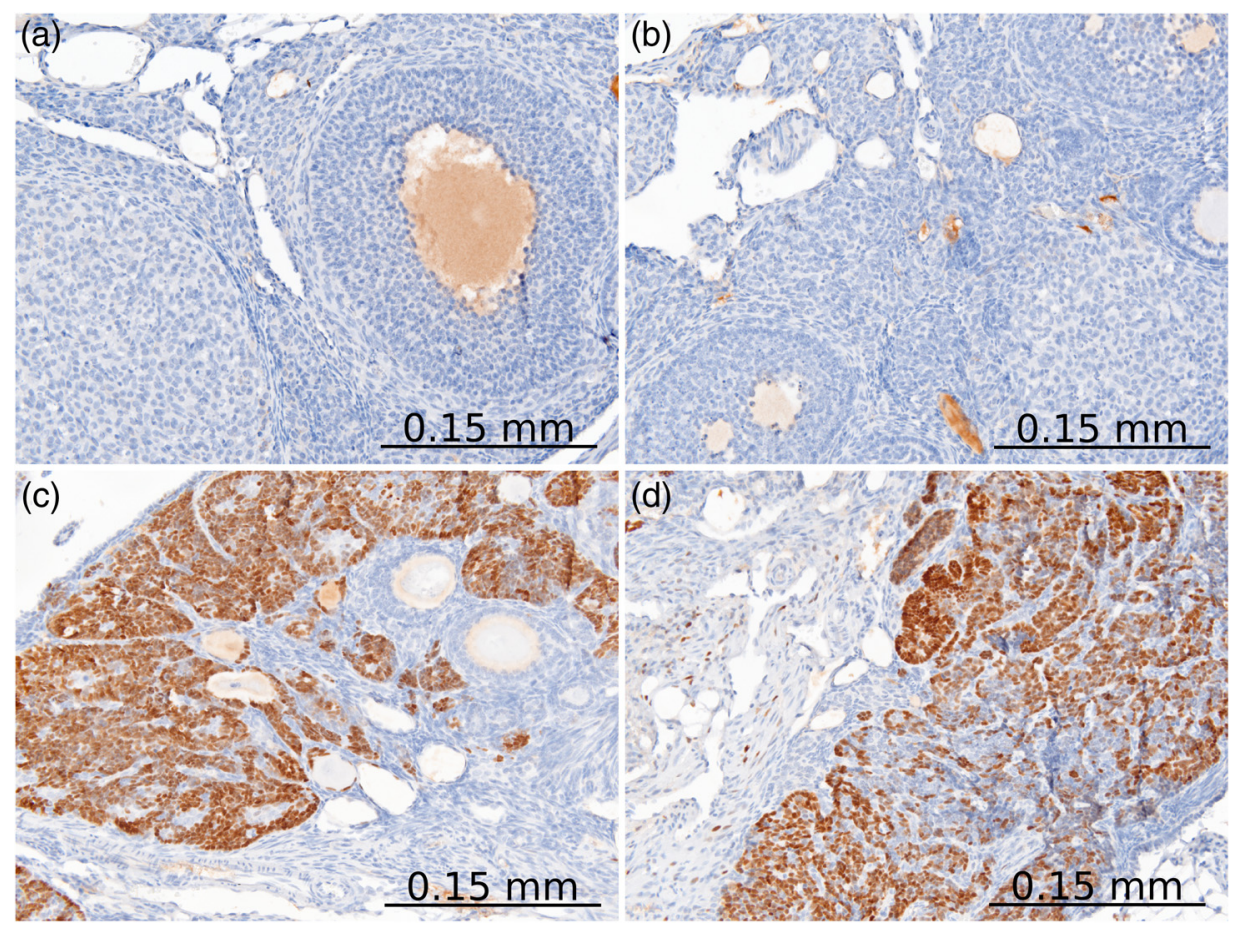

Fig. 2 TAg staining of an ovary at 40x; (a), (b) a WT mouse; (c), (d) a TAg mouse.
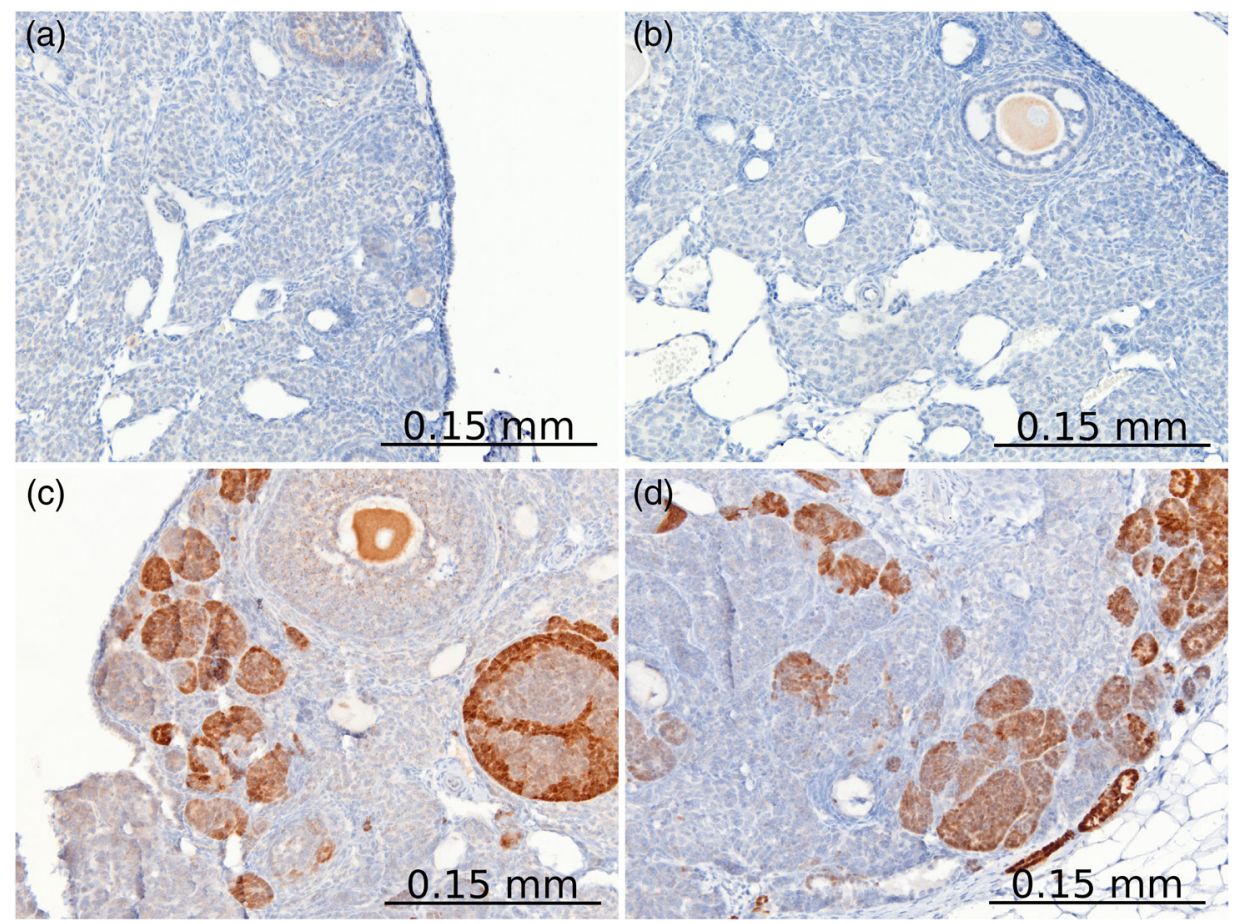

Fig. 3 PAX8 staining of an ovary at 40x; (a), (b) a WT mouse; (c), (d) a TAg mouse. 


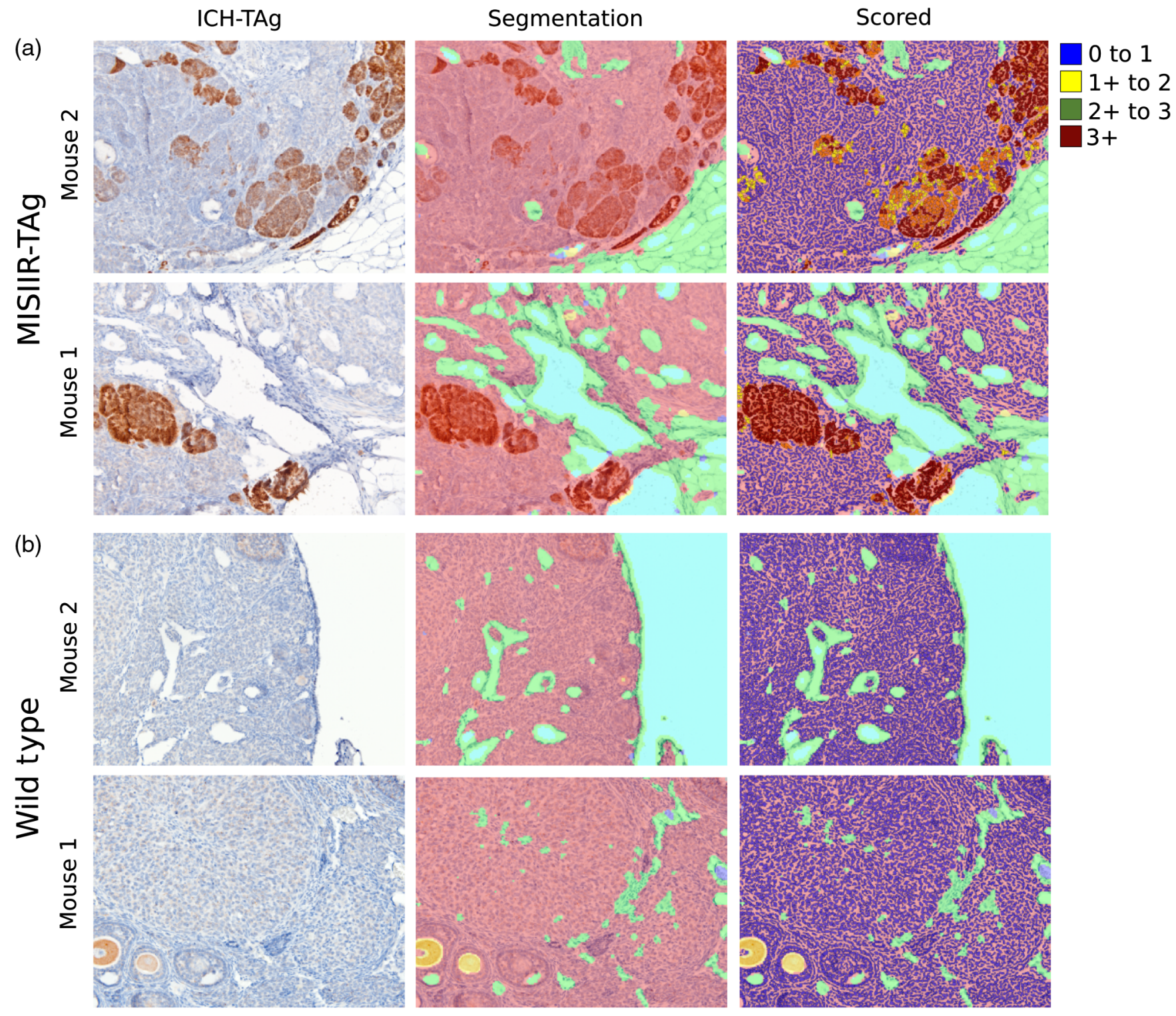

Fig. 4 Tissue segmentation and IHC stain scoring. The images shown depict sections of ovaries from 8-week sesame-oil-treated (a) MISIIR-TAg and (b) wild type mice that were subjected to IHC staining for TAg (brown staining) and counterstained with hematoxylin (blue). The same tissue sections were subjected to tissue segmentation to define ovary (pink), nonovary tissue (green), glass (blue), and junk (yellow). The ovary segment of the tissue sections were subjected to scoring of TAg staining in the nuclear compartment. The intensity of nuclear TAg staining was scored 0 to $3+$, where blue $=0$ (no staining), yellow $=1+$ to 2 , orange $=2+$ to 3 , and brown $=3+$.

some significant differences between treatments. Analysis of the $\%$ tumor, follicles, corpora lutea, and atretic follicles is included in Sec. 5 .

\subsection{Wide-Field Fluorescence Imaging}

Sample images for WFI of MMPSense are shown in Fig. 6 for 8-week wild type (a) and TAg (c) mice dosed with sesame oil. There is a demonstrable increase in the fluorescent signal for the TAg mouse. This is also observed for other experimental groups, such as 4-week mice dosed with VCD, as shown in Figs. 6(b) and 6(d). Results from the image analysis are summarized in Fig. 7. Consistent with our previous work, ${ }^{54}$ the signal generated by the MMPSense dye shows significant changes between wild type and TAg mice both in the ovaries and the oviducts. In addition, we see that the signal collected while using the FolateRSense dye is also significant for the oviduct between wild type and TAg mice. We also see that the fluorescence signal for MMPSense applied to the oviducts changes between mice treated with sesame oil and those treated with VCD at both 4 and 8 weeks of age. MMPs have been shown histologically to be relevant for human ovarian cancer, ${ }^{44,55}$ and targeting of the folate receptor has been demonstrated in human metastatic ovarian cancer in vivo. ${ }^{8,56}$ Therefore, we believe our results in the mouse model of early ovarian cancer are likely to also be relevant to a human population.

These results are consistent with expectations. We expect that there would be more MMPs with the presence of disease because cancer cells will need to break down the matrix to make space as they grow. The folate receptor is commonly 

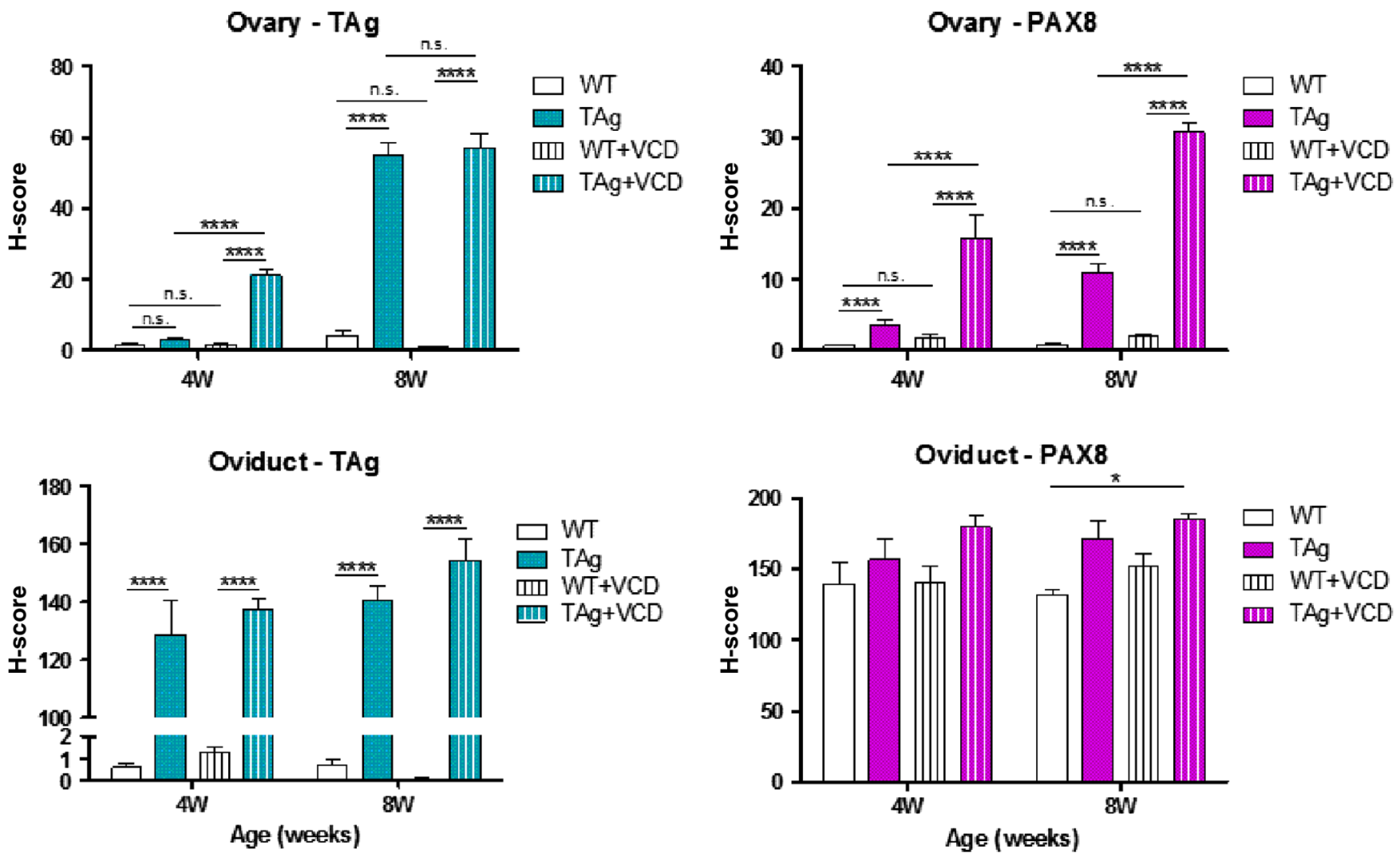

Fig. 5 Differences in $\mathrm{H}$-score between mouse groups for the oviduct and ovaries for both TAg staining and PAX8 staining.
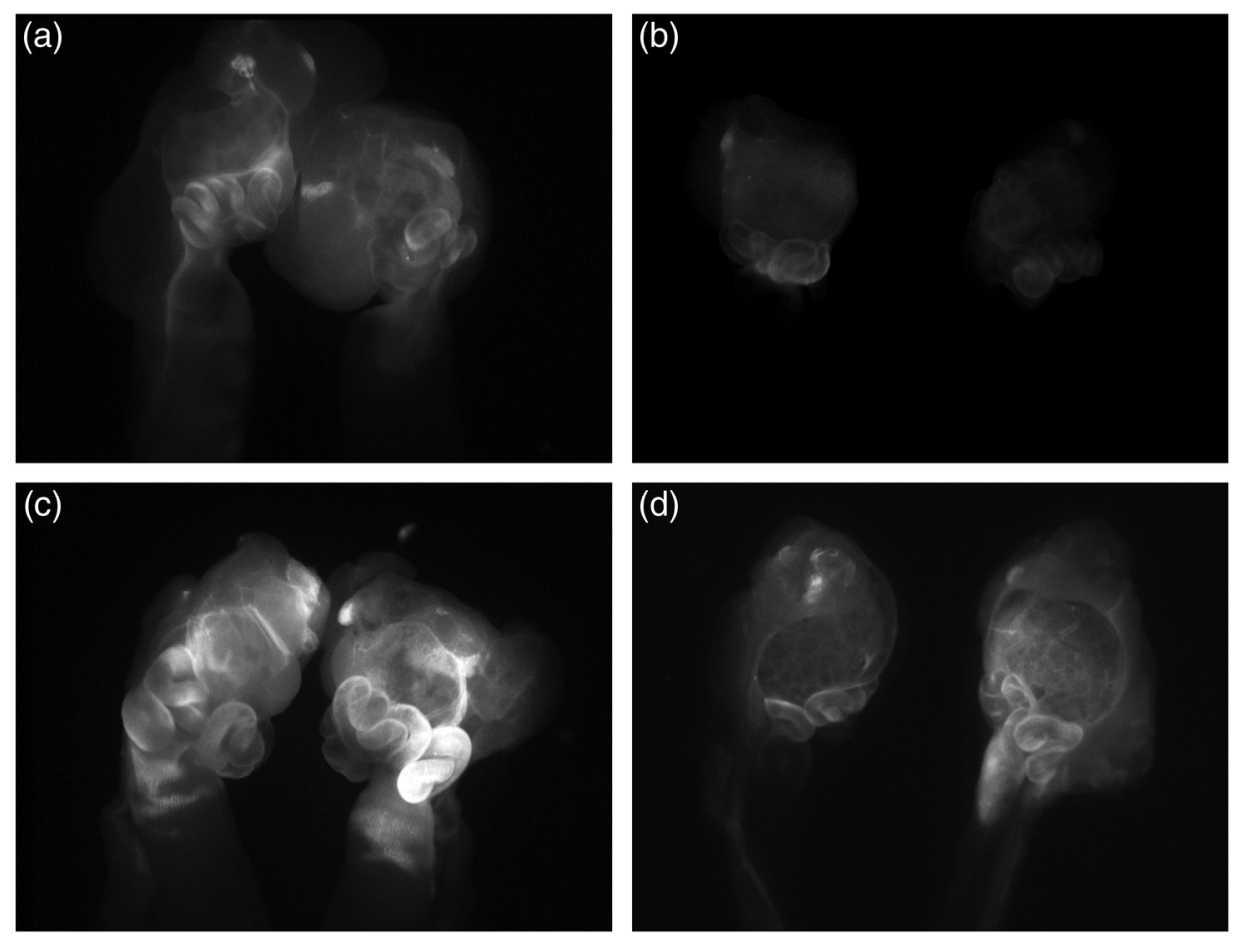

Fig. 6 Example images using WFI with MMPSense for the ovaries of (a) a wild type and (c) TAg mouse, both dosed with sesame oil at 8 weeks; (b) a wild type and (d) TAg mouse, both dosed with VCD at 4 weeks. Pathology indicated that the wild type mice were normal and the TAg mice had over $50 \%$ tumor cell percentage for both the ovaries and oviducts. 

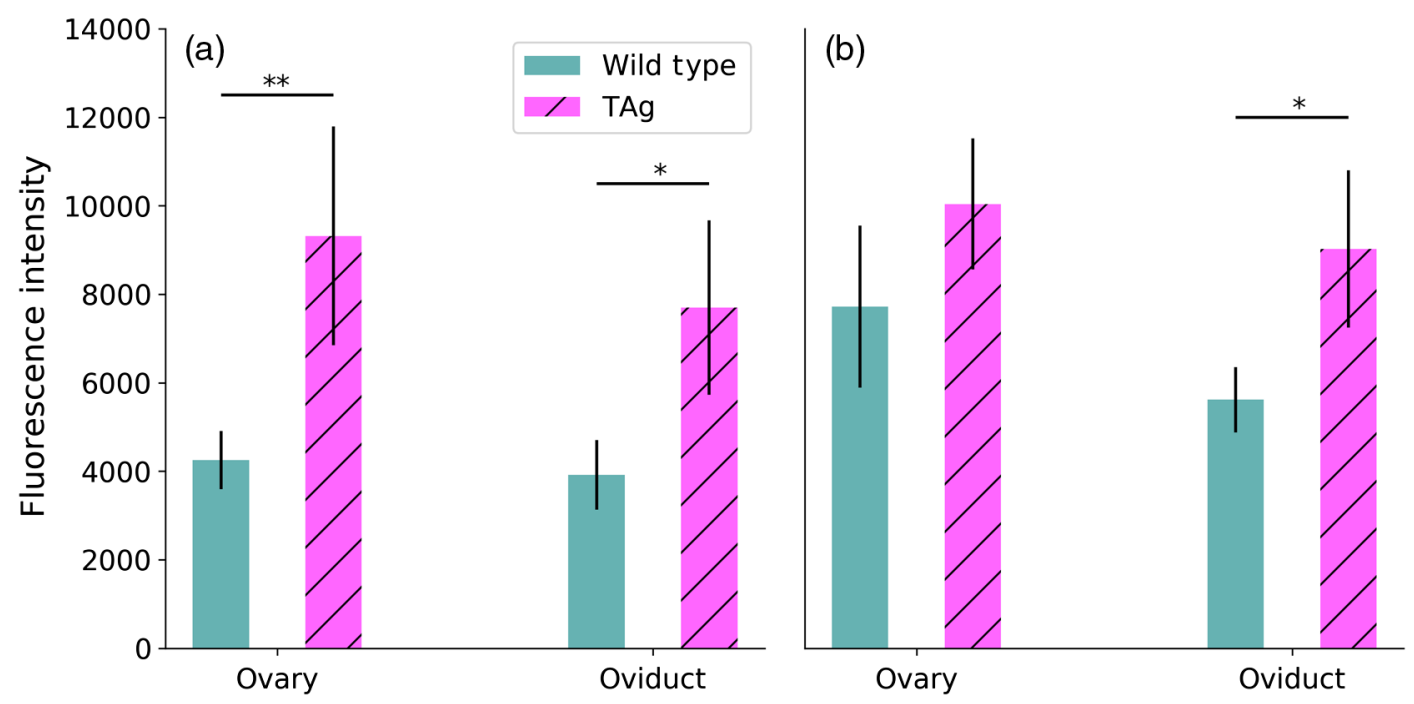

Fig. 7 Fluorescence signal collected for TAg and wild type mice at 8 weeks dosed with sesame oil with (a) MMPSense and (b) FolateRSense applied.

over-expressed in ovarian cancers, so we would expect that the TAg animals, which have developed cancer by 8 weeks, would have more folate receptors and hence more fluorescent signal from the FolateRSense.

\subsection{Multiphoton Images}

Representative SHG and 2PEF images for the oviducts and ovaries are shown in Fig. 8 for a wild type mouse at 8 weeks, as well as for a TAg mouse at 8 weeks, where histopathology analysis confirmed that $\sim 50 \%$ of the volume of the ovaries and oviducts was occupied by malignancy. In particular, the structure of the ovaries for both SHG and $2 \mathrm{PEF}$ is visibly more irregular compared with the wild type mouse. Furthermore, the collagen structure of the oviducts appears disordered in the TAg mouse compared with the wild type.

\subsection{Texture Analysis of Multiphoton Images}

Selected results for features from the GLCM analysis are shown in Fig. 9. Using the features to perform pairwise comparison tests between different mouse groups, multiple features are significant in all four imaging situations: SHG and 2PEF for both the ovaries and oviducts. Given the large number of features and comparisons, we select a subset for display. In the following section, however, we conducted an aggregated comparison between age, genotype, and treatment using a linear mixed model. The most sensitive features are correlation-based features of SHG and 2PEF imaging of the ovaries [Figs. 9(a) and 9(b)]. However, we also find that other complementary features such as variance and entropy-based features for SHG and 2PEF imaging of the oviducts [Figs. 9(c) and 9(d)] provide high statistical significance for separating groups. In general, age tends to yield the highest differences between features, indicating that this may be a particularly challenging effect to overcome. This dependence on age seems consistent with tumor phenotype, both in terms of organ and age. In this model, there are neoplastic changes in the oviduct at 4 and 8 weeks. At 4 weeks, some TAg+ tumor cells are observed in the ovary, but the tissue is predominantly normal. At 8 weeks, the number of TAg+ cells has increased to a significant degree.
Select frequency features are shown for SHG and $2 \mathrm{PEF}$ imaging for the ovaries [Figs. 10(a) and 10(b)] and the oviducts [Figs. 10(c) and 10(d)]. Not all statistical comparisons are shown for the sake of legibility; however, Tables 3-5 in Sec. 6 summarize whether the individual features are significant for each comparison. The results show that the parameters alpha and beta provide significant power for differentiating between experimental groups. The constant offset parameter gamma does not show statistical significance between experimental groups.

\subsection{Variations by Group}

The results using a linear mixed model to determine significance for texture features in MPM images are shown in Tables 3-5 in Sec. 6 for age, genotype, and VCD dosing, respectively. The second of these groups has the most diagnostic relevance, as pathology indicates all TAg mice develop ovarian cancer by 8 weeks of age. $2 \mathrm{PEF}$ imaging of the ovaries produces six features that have high statistical significance for modeling the genotype. The confounding effects of age and treatment may reduce the fidelity of the feature discrimination for genotype. However, assessing the influence of age and reproductive status is more realistic, as cancer can develop at any stage of life, though the highest risk does occur in older women who are postmenopausal. SHG imaging shows no significant features for differentiating genotype; however, several features are sensitive to both VCD dosing and age. Previous studies have shown that VCD dosing is a good analog for menopausal status, with over $50 \%$ of 8 -week mice dosed with VCD having undergone ovarian failure or impending ovarian failure, compared with less than $20 \%$ of 8 -week mice that are dosed with sesame oil. ${ }^{57}$

Although these results are encouraging, note that the statistical test used here (Wald test) assesses whether each texture feature is significant in building a linear model of the experimental variable using the features as explanatory variables. This is not equivalent to an ANOVA test, which is used for individual group comparisons, e.g., those shown in Figs. 9 and 10. However, quantitative tissue classification is often accomplished using a linear model, as we demonstrate in Sec. 3.6. Hence, using the linear model approach shown here is relevant for assessing diagnostic potential. 


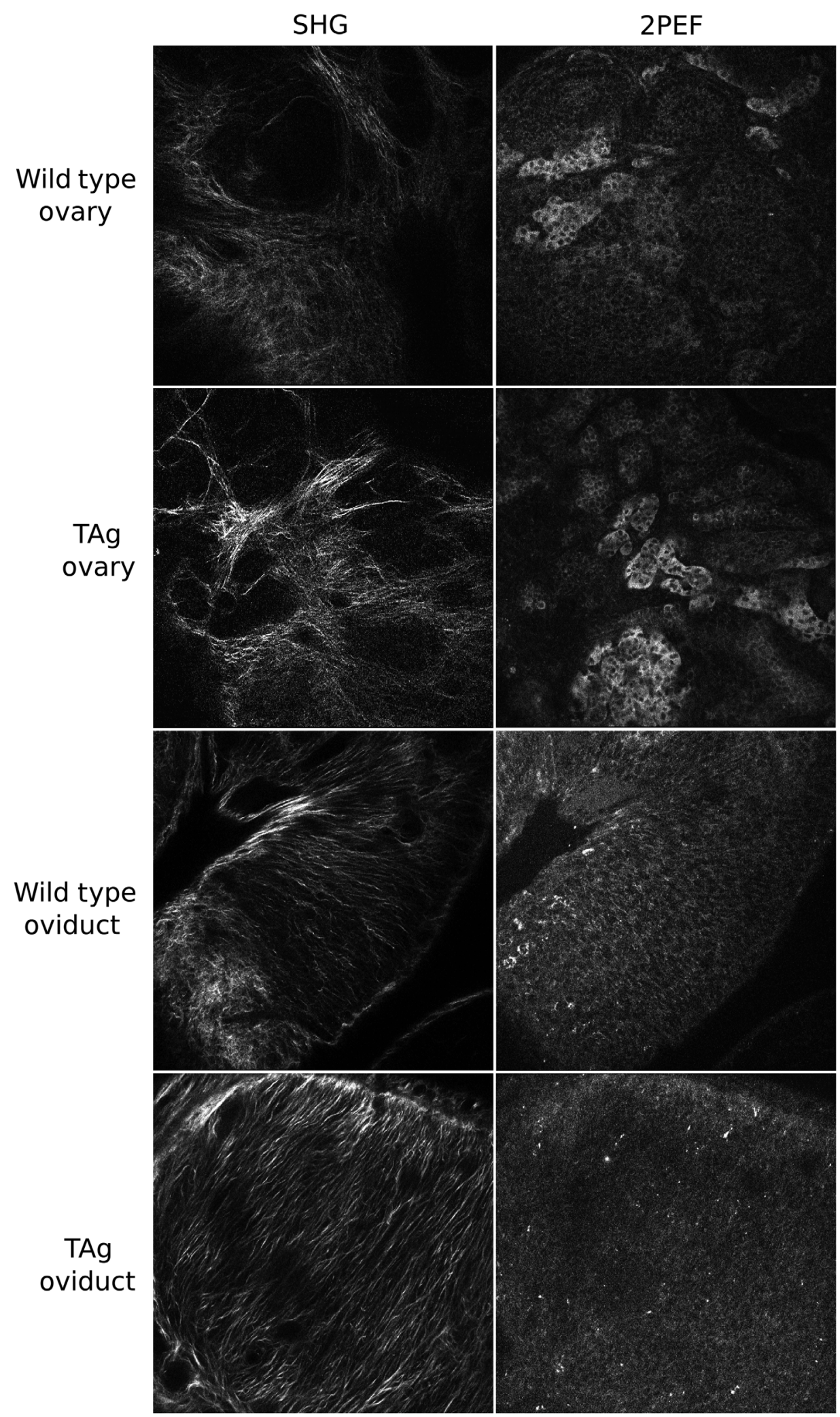

Fig. 8 Example images using SHG and 2PEF for the ovaries and oviducts collected for a wild type and TAg mice. Pathology indicated that the wild type mouse was normal and the TAg mouse had over $50 \%$ tumor cell percentage for both the ovaries and oviducts.

Although histology indicates that genotype is a good proxy for disease, we did not observe predictable trends in the features as the degree of tumor percentage increases in the mice. The limited sample size poses a challenge for detecting trends in the observed results; for example, only six mice exhibited more than $10 \%$ tumor percentage. Furthermore, only portions of the organs contained tumor cells; given that MPM does not cover the entire organ, the imaging data may contain both healthy and diseased 

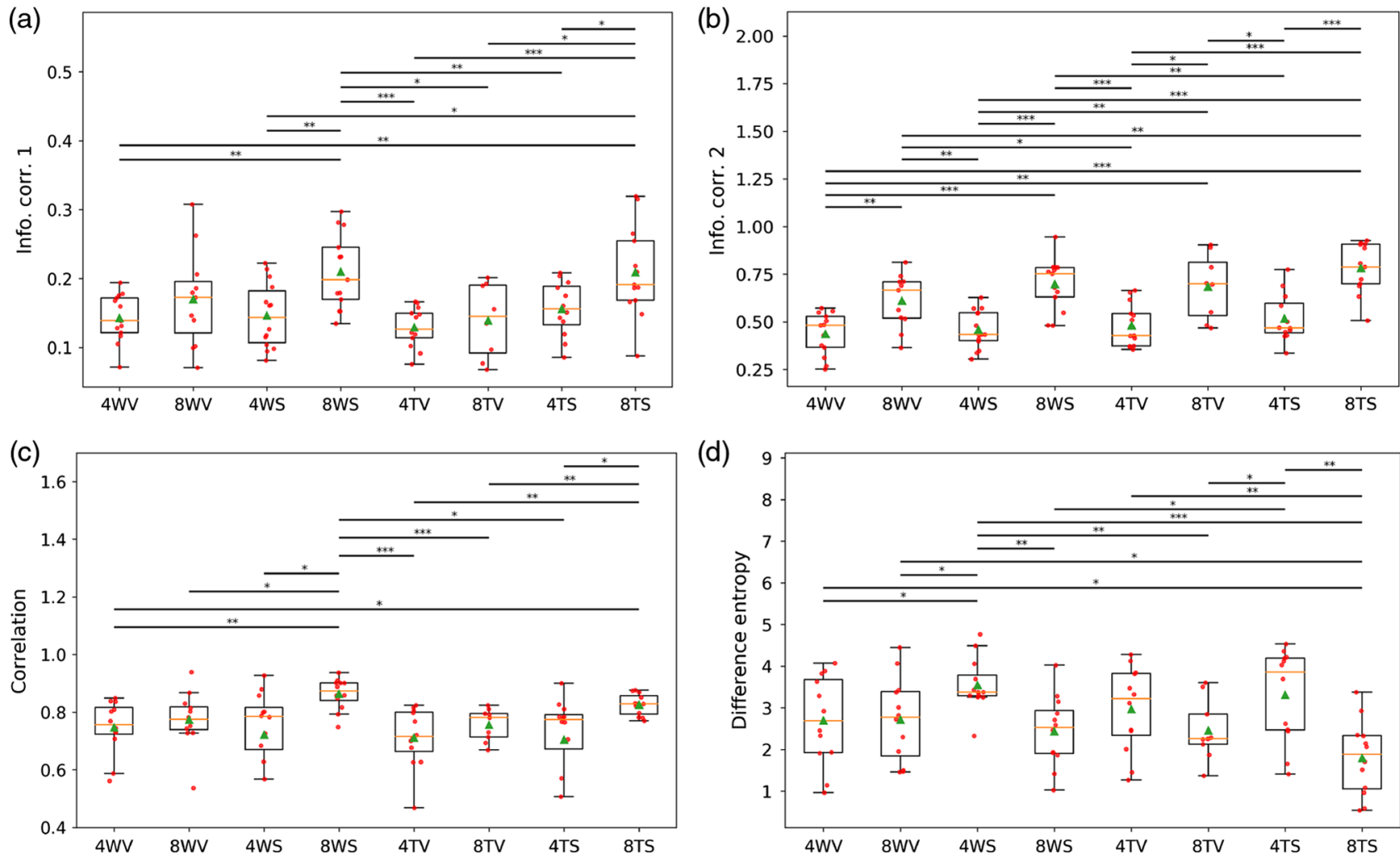

Fig. 9 Selected GLCM texture features for (a) SHG ovaries, (b) 2PEF ovaries, (c) SHG oviduct, and (d) 2PEF oviduct. We see wide significance for using GLCM features to differentiate between different experimental groups. [W, wild type; T, Tag; S, treated with vehicle (sesame oil); and V, treated with VCD].

(a)

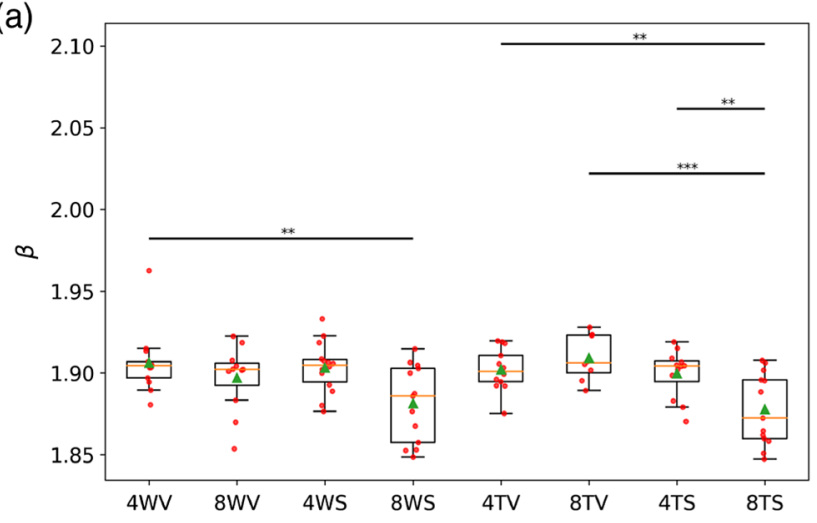

(c)

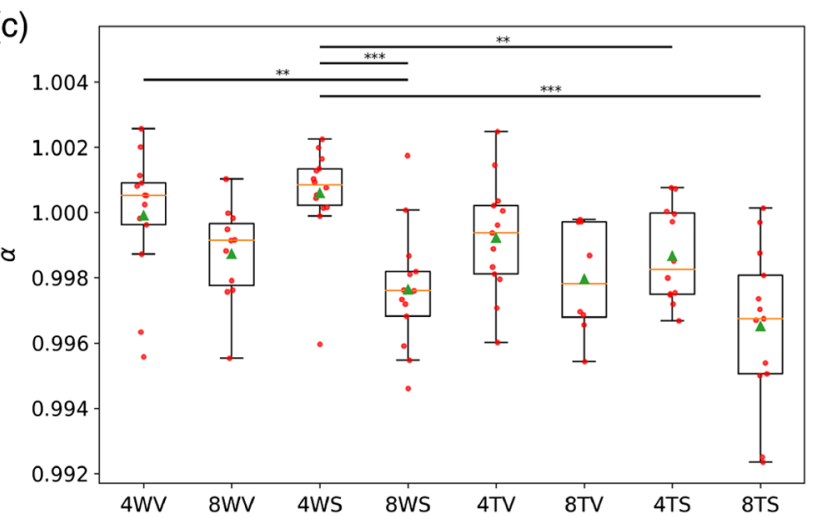

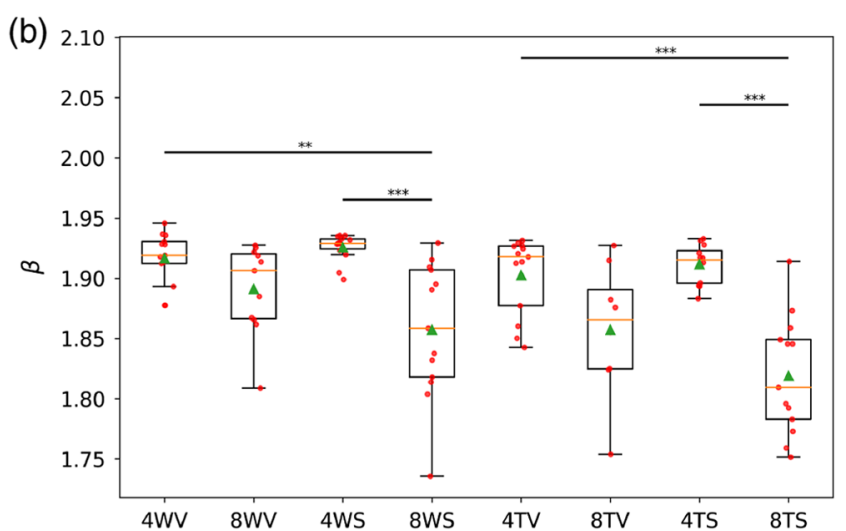

(d)

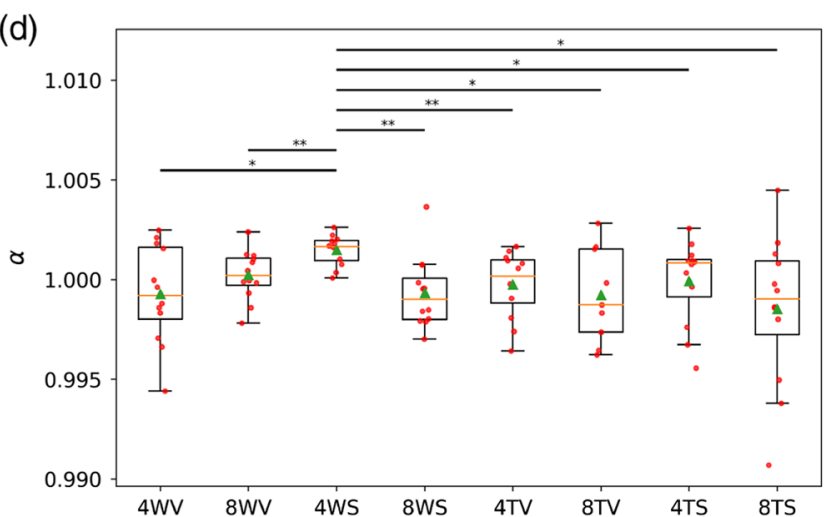

Fig. 10 Selected frequency-based texture features for (a) SHG ovaries, (b) 2PEF ovaries, (c) SHG oviduct, and (d) 2PEF oviduct. [W, wild type; T, Tag; S, treated with vehicle (sesame oil); V, treated with VCD; and $4 / 8$, weeks of age]. 
tissue. However, there may still be early changes in the surrounding healthy tissue that could be reflected using MPM. Conducting a more in-depth investigation into whether trends exist in the features as a function of disease progression and tumor cell percentage is an objective for future work.

\subsection{Classification}

The classification results are summarized in Table 2, where the average classification accuracy for each type of comparison (age, genotype, and treatment) is shown for different feature sets (SHG and 2PEF images of the ovary and oviduct). This classification is done using MPM features only. We see that combining the four individual feature sets into a single large set yields overall higher classification accuracy, with $87.50 \%, 66.66 \%$, and $62.50 \%$, for age, genotype, and treatment, respectively. This is not surprising, as it was shown in the individual comparison that the different modalities are sensitive to different types of changes. This finding validates the expectation that combining morphological and functional information can enhance accuracy for tissue characterization. We also see that only a small subset of features is required to achieve maximum classification accuracy: in each case, six features or less yielded

Table 2 Average classification accuracies for age, genotype, and treatment group comparisons, as well as optimal number of features to achieve this accuracy. The results are shown for individual feature sets corresponding to each imaging modality and organ, as well as using all features together.

\begin{tabular}{lcccc}
$\begin{array}{l}\text { Features } \\
\text { selected }\end{array}$ & $\begin{array}{c}\text { Optimal \# } \\
\text { of features }\end{array}$ & Age & Genotype & Treatment \\
\hline SHG ovary & 6 & 0.6490 & 0.3933 & 0.5185 \\
PEF ovary & 4 & 0.8099 & 0.6289 & 0.4593 \\
SHG oviduct & 6 & 0.6547 & 0.5079 & 0.5178 \\
PEF oviduct & 5 & 0.6696 & 0.4206 & 0.5937 \\
All & 5 & 0.8750 & 0.6666 & 0.6250 \\
\hline
\end{tabular}

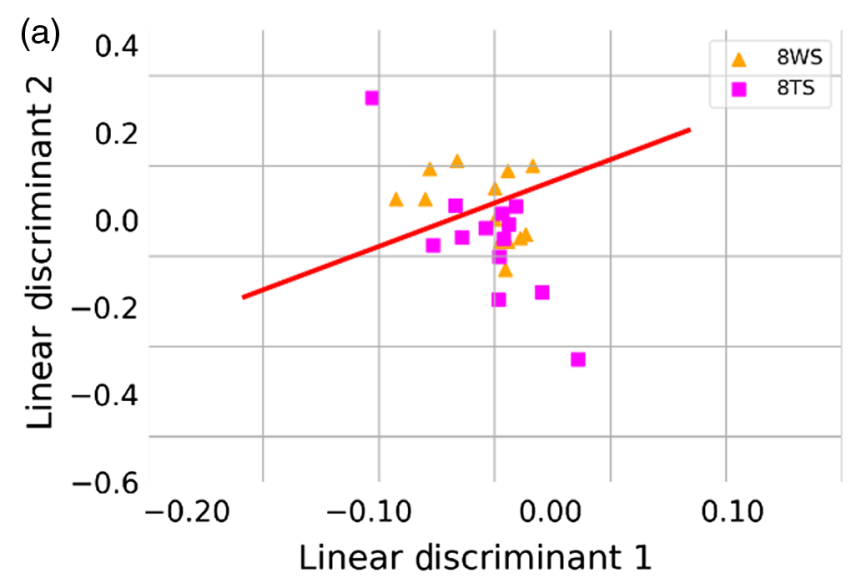

the best accuracy. This validates the expectation that some features are only relevant for separation when combined with other complementary features, further motivating the use of dimension reduction techniques such as LDA or principal component analysis.

Example results for the LDA are shown in Fig. 11 for a comparison between genotype [Fig. 11(a)] and age [Fig. 11(b)]. Although encouraging, a linear model is limited in its capabilities and better performance may be accessible when using more complex techniques such as machine learning. Generally, with a small number of samples, the complexity of the model is likewise limited; however, extending the classification to a more advanced algorithm is a focus of ongoing work. Additionally, future work is focused on applying these modalities (MPM and WFI) to probe tissue changes in the oviducts that may occur with the early onset of ovarian cancer in humans.

\section{Conclusion}

In this paper, we assessed the potential of MPM and fluorescence imaging for evaluating ovarian tissue health. We imaged a transgenic mouse model that spontaneously developed ovarian cancer in vivo using both SHG and 2PEF imaging, as well as WFI to target dyes that bound to the folate receptor and MMPs. Using texture analysis of multiphoton images based on the GLCM as well as features describing the frequency content of these images, we showed that it is possible to differentiate between experimental groups (age, genotype, and reproductive status) with high statistical significance $(p<0.001)$. We also see significant changes in the signal collected by wide-field fluorescent imaging between experimental groups $(p<0.01)$. We then used these features to build a classification algorithm using LDA, showing that we can classify different ages, genotypes, and treatments with accuracies of $87.50 \%, 66.66 \%$, and $62.50 \%$, respectively. Although these results are promising, the next steps include implementing a more complex a classification scheme using methods such as machine learning. Furthermore, we used the TAg genotype as a proxy for disease. Although histology showed that all TAg mice developed some degree of disease by 8 weeks, conducting a further experiment with histopathology results is of particular interest to determine whether trends can be established between the tissue changes observed and tumor percentage.

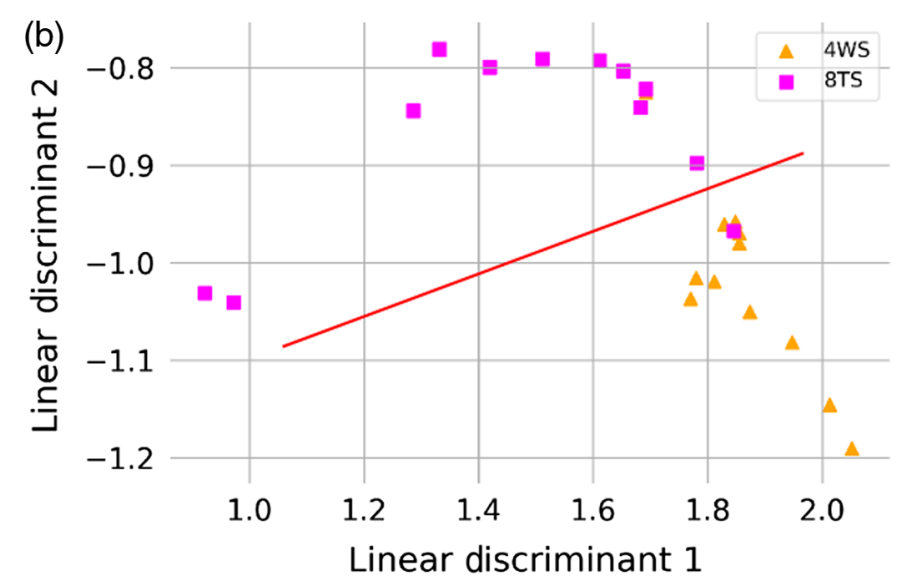

Fig. 11 An example of separating two groups based on the first two linear discriminants using features from (a) SHG imaging of the ovaries and (b) 2PEF imaging of the ovaries. (W, wild type; T, Tag; and S, treated with sesame oil). 


\section{Appendix I: Additional Histological Analysis}

This appendix contains histological results for the mouse tissues. Figure 12 illustrates the assessment for the different experimental groups, including \% tumour cells [Fig. 12(a)], follicles [Fig. 12(b)], corpora lutea [Fig. 12(c)], and \% atretic follicles [Fig. 12(d)].

MMP sense treated

(a)

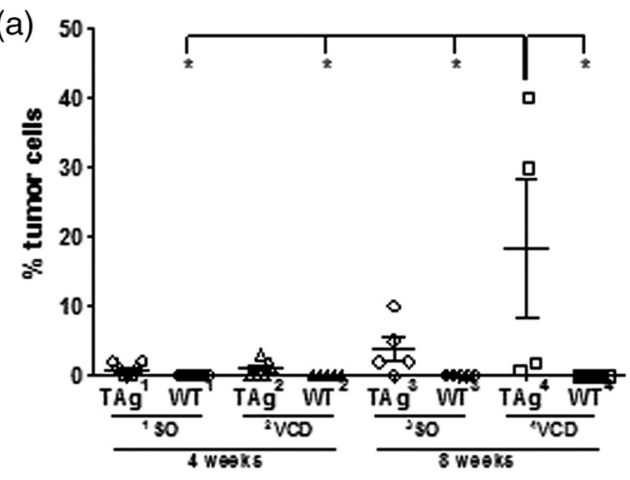

(b)

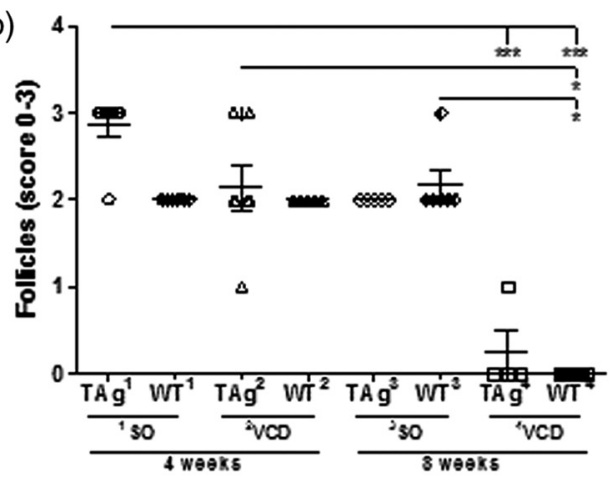

(c)

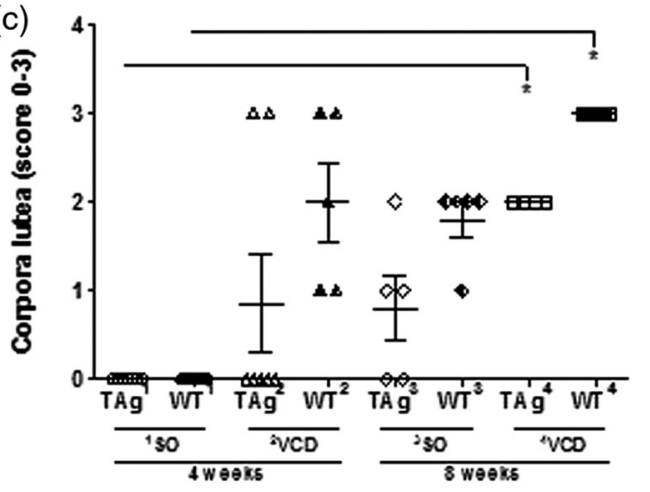

(d)

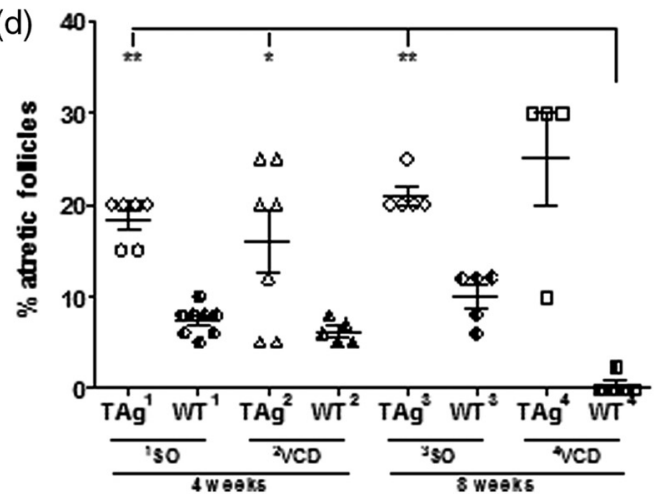

\section{Folate sense treated}
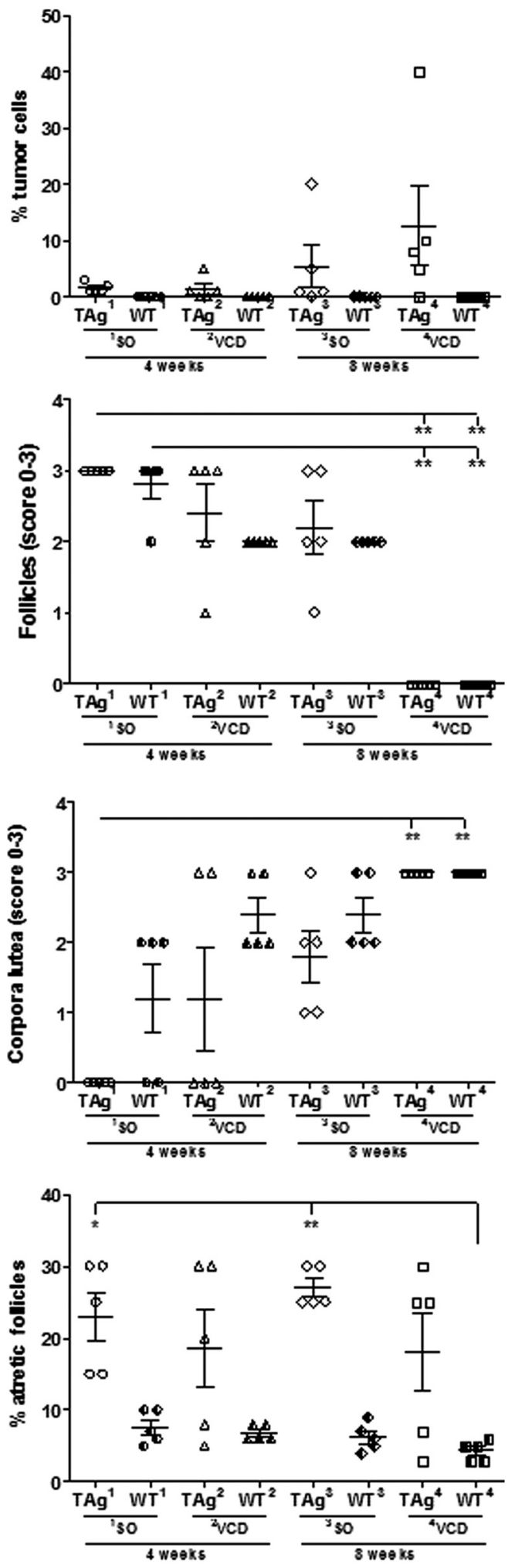

Fig. 12 Histology results for complete mouse study, organized by mice treated with MMPSense (first column) and FolateRSense (second column); ${ }^{1} \mathrm{SO}$, treated with sesame oil; ${ }^{2} \mathrm{VCD}$, treated with VCD; ${ }^{3} \mathrm{WT}$, wild type mice; and ${ }^{4} \mathrm{TAg}$, TAg transgenic mice. The analysis indicated $\%$ (a) tumor cell, (b) follicles, (c) corpora lutea, and (d) \% atretic follicles. 


\section{Appendix II: Feature Significance for Group Comparisons}

This appendix contains the individual performance for each texture feature when differentiating between experimental groups using a linear mixed model. Table 3 shows results for differentiating by age group, Table 4 for differentiating by genotype, and Table 5 for separating based on treatment status.

Table 3 Significance of features for each imaging modality and organ for differentiating mice by age using a linear mixed model.

\begin{tabular}{|c|c|c|c|c|}
\hline \multirow[b]{2}{*}{ Feature } & \multicolumn{2}{|c|}{ Ovaries } & \multicolumn{2}{|c|}{ Oviducts } \\
\hline & SHG & 2PEF & SHG & 2PEF \\
\hline Angular second moment & - & - & ** & - \\
\hline Contrast & - & * & - & - \\
\hline Correlation & - & $* \star *$ & $* * *$ & * \\
\hline Sum of squares: variance & - & - & - & - \\
\hline Inverse difference moment & - & - & - & ** \\
\hline Sum average & - & - & - & ** \\
\hline Sum entropy & - & * & - & - \\
\hline Sum variance & - & - & * & ** \\
\hline Entropy & - & - & - & $\star *$ \\
\hline Difference variance & - & * & - & - \\
\hline Difference entropy & - & - & - & $* \star *$ \\
\hline Info. measure of correlation 1 & * & - & $* \star *$ & $* \star *$ \\
\hline Info. measure of correlation 2 & - & 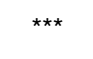 & $* \star *$ & - \\
\hline$\alpha$ & - & $\star \star * *$ & - & - \\
\hline$\beta$ & $\star \star$ & 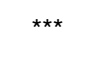 & $\star \star \star \star$ & - \\
\hline$\gamma$ & - & - & - & - \\
\hline
\end{tabular}

Table 4 Significance of features for each imaging modality and organ for differentiating mice by genotype using a linear mixed model.

\begin{tabular}{lccccc}
\hline & \multicolumn{2}{c}{ Ovaries } & & \multicolumn{2}{c}{ Oviducts } \\
\cline { 2 - 3 } \cline { 5 - 6 } Feature & SHG & 2PEF & & SHG & 2 PEF \\
\hline Angular second moment & - & - & & - & - \\
Contrast & - & - & & - & - \\
Correlation & - & $* *$ & & - & $*$ \\
Sum of squares: variance & - & $*$ & & - & - \\
Inverse difference moment & - & - & - & - \\
\hline
\end{tabular}

Table 4 (Continued).

\begin{tabular}{lccccc}
\hline & \multicolumn{2}{c}{ Ovaries } & & \multicolumn{2}{c}{ Oviducts } \\
\cline { 2 - 3 } \cline { 5 - 6 } Feature & SHG & 2PEF & & SHG & 2 PEF \\
\hline Sum average & - & $*$ & & - & - \\
Sum entropy & - & $*$ & & - & - \\
Sum variance & - & - & & - & - \\
Entropy & - & - & & - & - \\
Difference variance & - & - & & - & - \\
Difference entropy & - & - & & - & - \\
Info. measure of correlation 1 & $*$ & - & - & - \\
Info. measure of correlation 2 & $*$ & - & - & - \\
$\alpha$ & - & $* *$ & - & - \\
$\beta$ & - & $* *$ & - & - \\
$\gamma$ & & & & & - \\
\hline
\end{tabular}

Table 5 Significance of features for each imaging modality and organ for differentiating mice by VCD dosing using a linear mixed model.

\begin{tabular}{|c|c|c|c|c|}
\hline \multirow[b]{2}{*}{ Feature } & \multicolumn{2}{|c|}{ Ovaries } & \multicolumn{2}{|c|}{ Oviducts } \\
\hline & SHG & 2PEF & SHG & 2PEF \\
\hline Angular second moment & - & - & - & - \\
\hline Contrast & - & - & - & - \\
\hline Correlation & ** & * & - & - \\
\hline Sum of squares: variance & - & - & - & - \\
\hline Inverse difference moment & - & - & - & - \\
\hline Sum average & - & - & - & - \\
\hline Sum entropy & - & - & - & - \\
\hline Sum variance & - & - & - & - \\
\hline Entropy & - & - & - & - \\
\hline Difference variance & - & - & - & - \\
\hline Difference entropy & - & - & - & - \\
\hline Info. measure of correlation 1 & ** & * & - & - \\
\hline Info. measure of correlation 2 & $\star \star$ & * & - & - \\
\hline$\alpha$ & - & - & - & - \\
\hline$\beta$ & - & - & * & - \\
\hline$\gamma$ & - & - & - & - \\
\hline
\end{tabular}




\section{Disclosures}

We have no relevant financial interests and no other potential conflicts of interest to disclose.

\section{Acknowledgments}

This work was supported by the National Science Foundation Graduate Research Fellowship Program under Grant No. DGE-1143953, the National Institutes of Health under National Cancer Institute (Grant No. 1R01CA195723), and the shared resources of the University of Arizona Cancer Center (Grant No. 3P30CA023074). We also would like to acknowledge the support of the Theresa F. Jennings Memorial Scholarship. Any opinions, findings, and conclusions or recommendations expressed in this paper are those of the authors and do not necessarily reflect the views of the National Science Foundation. This work performed at Fox Chase Cancer Center (FCCC) in Dr. Connolly's Laboratory was additionally supported by the FCCC Core (Grant No. NCI P30 CA006927) and the FCCC Biosample Repository Facility and Histopathology Facility. Some results presented in this paper have been previously published in the proceedings of the SPIE conference, "Diseases in the Breast and Reproductive System V," edited by M.C. Skala, P.J. Campangola, and D.M. Roblyer. ${ }^{58}$ We gratefully acknowledge the technical support of Ms. Chao Wu of the Fox Chase Cancer Center Biosample Repository Facility for assistance with the Vectra Multispectral Imaging Analysis.

\section{References}

1. R. L. Siegel, K. D. Miller, and A. Jemal, "Cancer statistics, 2019," $C A$ Cancer J. Clin. 69(1), 7-34 (2019).

2. A. M. Noone et al., Eds., "SEER Cancer Statistics Review," 1975-2015, National Cancer Institute, 2017, https://seer.cancer.gov/csr/1975_2015/ (April 2018).

3. C. Maringe et al., "Stage at diagnosis and ovarian cancer survival: evidence from the international cancer benchmarking partnership," Gynecol. Oncol. 127(1), 75-82 (2012).

4. I. J. Jacobs et al., "Ovarian cancer screening and mortality in the UK collaborative trial of ovarian cancer screening (UKCTOCS): a randomised controlled trial," Lancet 387(10022), 945-956 (2016).

5. K. Carlson, S. Skates, and D. Singer, "Screening for ovarian cancer," Ann. Intern. Med. 124, 124-132 (1994).

6. V. A. Moyer, "Screening for ovarian cancer: U.S. preventive services task force reaffirmation recommendation statement," Ann. Intern. Med. 157(12), 900-904 (2012).

7. R. George et al., "Clinical research device for ovarian cancer detection by optical spectroscopy in the ultraviolet C-visible," J. Biomed. Opt 15(5), 057009 (2010).

8. G. M. Van Dam et al., "Intraoperative tumor-specific fluorescence imaging in ovarian cancer by folate receptor- $\alpha$ targeting: First in-human results," Nat. Med. 17(10), 1315-1319 (2011).

9. A. A. Tanbakuchi et al., "In vivo imaging of ovarian tissue using a novel confocal microlaparoscope," Am. J. Obstet. Gynecol. 202(1), 90.e1-90.e9 (2010).

10. T. Wang et al., "Characterization of ovarian tissue based on quantitative analysis of photoacoustic microscopy images," Biomed. Opt. Express 4(12), 2763-2768 (2013).

11. T. Sawyer et al., "Three-dimensional texture analysis of optical coherence tomography images of ovarian tissue," Phys. Med. Biol. 63(23), 235020 (2018).

12. J. M. Watson et al., "Two-photon excited fluorescence imaging of endogenous contrast in a mouse model of ovarian cancer," Lasers Surg. Med. 45, 155-166 (2013).

13. W. A. Welge et al., "Diagnostic potential of multimodal imaging of ovarian tissue using optical coherence tomography and second-harmonic generation microscopy," J. Med. Imaging 1(2), 025501 (2014).
14. P. J. Campagnola and L. M. Loew, "Second-harmonic imaging microscopy for visualizing biomolecular arrays in cells, tissues and organisms," Nat. Biotechnol. 21(11), 1356-1360 (2003).

15. R. Richards-Kortum and E. Sevick-Muraca, "Quantitative optical spectroscopy for tissue diagnosis," Аnnu. Rev. Phys. Chem. 47, 555-606 (1996).

16. J. R. Lakowicz, Principles of Fluorescence Spectroscopy, Springer, Boston, Massachusetts (2006).

17. W. Denk, J. H. Strickler, and W. W. Webb, "Two-photon laser scanning fluorescence microscopy," Science 248(4951), 73-76 (1990).

18. A. Ustione and D. W. Piston, "A simple introduction to multiphoton microscopy," J. Microsc. 243(3), 221-226 (2011).

19. R. Niesner et al., "The power of single and multibeam two-photon microscopy for high-resolution and high-speed deep tissue and intravital imaging," Biophys. J. 93(7), 2519-2529 (2007).

20. E. E. Hoover and J. A. Squier, "Advances in multiphoton microscopy technology," Nat. Photonics 7(2), 93-101 (2013).

21. G. Toffoli et al., "Overexpression of folate binding protein in ovarian cancers," Int. J. Cancer 74(2), 193-198 (1997).

22. C. Gialeli, A. D. Theocharis, and N. K. Karamanos, "Roles of matrix metalloproteinases in cancer progression and their pharmacological targeting," FEBS J. 278(1), 16-27 (2011).

23. M. Strupler et al., "Second harmonic imaging and scoring of collagen in fibrotic tissues," Opt. Express 15(7), 4054-4065 (2007).

24. D. C. S. Tai et al., "Fibro-C-Index: comprehensive, morphology-based quantification of liver fibrosis using second-harmonic generation and two-photon microscopy," J. Biomed. Opt. 14(4), 044013 (2009).

25. R. Cicchi et al., "Scoring of collagen organization in healthy and diseased human dermis by multiphoton microscopy," J. Biophotonics 3(1-2), 34-43 (2010).

26. J. Adur et al., "Recognition of serous ovarian tumors in human samples by multimodal nonlinear optical microscopy," J. Biomed. Opt. 16(9), 096017 (2011).

27. R. Ambekar et al., "Quantifying collagen structure in breast biopsies using second-harmonic generation imaging," Biomed. Opt. Express 3(9), 2021-2035 (2012).

28. S. Plotnikov et al., "Optical clearing for improved contrast in secondharmonic generation imaging of skeletal muscle," Biophys. J. 90(1), 328-339 (2006).

29. K. Tilbury et al., "Second-harmonic generation microscopy analysis of extracellular matrix changes in human idiopathic pulmonary fibrosis," J. Biomed. Opt. 19(8), 086014 (2014).

30. M. W. Conklin et al., "Aligned collagen is a prognostic signature for survival in human breast carcinoma," Am. J. Pathol. 178(3), 1221-1232 (2011).

31. B. L. Wen et al., "Texture analysis applied to second-harmonic generation image data for ovarian cancer classification," J. Biomed. Opt. 19(9), 096007 (2014).

32. N. D. Kirkpatrick, M. A. Brewer, and U. Utzinger, "Endogenous optical biomarkers of ovarian cancer evaluated with multiphoton microscopy," Cancer Epidemiol. Biomarkers Prev. 16(10), 2048-2057 (2007).

33. R. M. Williams et al., "Strategies for high resolution imaging of epithelial ovarian cancer by laparoscopic nonlinear microscopy," Transl. Oncol. 3(3), 181-194 (2010).

34. O. Nadiarnykh et al., "Alterations of the extracellular matrix in ovarian cancer studied by second-harmonic generation imaging microscopy," BMC Cancer 10, 94 (2010).

35. D. Granot et al., "In vivo imaging of the systemic recruitment of fibroblasts to the angiogenic rim of ovarian carcinoma tumors," Cancer Res. 67(19), 9180-9189 (2007).

36. R. Haralick, K. Shanmugan, and I. Dinstein, "Textural features for image classification," IEEE Trans. Syst. Man. Cybern. Syst. SMC-3, 610-621 (1973).

37. R. M. Haralick, "Statistical and structural approaches to texture," Proc. IEEE 67(5), 786-804 (1979).

38. D. C. Connolly et al., "Female mice chimeric for expression of the simian virus $40 \mathrm{TAg}$ under control of the MISIIR promoter develop epithelial ovarian cancer," Cancer Res. 63(6), 1389-1397 (2003).

39. H. Hensley et al., "Magnetic resonance imaging for detection and determination of tumor volume in a genetically engineered mouse model of ovarian cancer," Cancer Biol. Ther. 6(11), 1717-1725 (2007).

40. B. A. Quinn et al., "Development of a syngeneic mouse model of epithelial ovarian cancer," J. Ovarian Res. 3(1), 24 (2010). 
41. M. J. Romero-Aleshire et al., "Loss of ovarian function in the VCD mouse-model of menopause leads to insulin resistance and a rapid progression into the metabolic syndrome," Am. J. Physiol. Regul. Integr. Comp. Physiol. 297(3), R587-R592 (2009).

42. J. M. Watson et al., "Multi-modality optical imaging of ovarian cancer in a post-menopausal mouse model," Proc. SPIE 7890, 78900W (2011).

43. K. R. Kalli et al., "Folate receptor alpha peptide vaccine generates immunity in breast and ovarian cancer patients," Clin. Cancer Res. 24(13), 3014-3025 (2018).

44. L. Al-Alem and T. E. Curry, "Ovarian cancer: involvement of the matrix metalloproteinases," Reproduction 150(2), R55-R64 (2015).

45. R. Gabbasov et al., "NEDD9 promotes oncogenic signaling, a stem/ mesenchymal gene signature, and aggressive ovarian cancer growth in mice," Oncogene 37, 4854-4870 (2018).

46. W. Rasband, ImageJ, U. S. National Institutes of Health, Bethesda, Maryland (2012).

47. C. A. Lingley-Papadopoulos et al., "Computer recognition of cancer in the urinary bladder using optical coherence tomography and texture analysis," J. Biomed. Opt. 13(2), 024003 (2008).

48. C. St-Pierre et al., "Dimension reduction technique using a multilayered descriptor for high-precision classification of ovarian cancer tissue using optical coherence tomography," J. Med. Imaging 4(4), 041306 (2017).

49. R. O. Duda, P. E. Hart, and D. G. Stork, Pattern Classification, 2 ed., Wiley, New York (2001).

50. M. Welling, "Fisher linear discriminant analysis," Science 1(2), 1-3 (2009).

51. G.-C. Lin et al., "Computerized medical imaging and graphics automated classification of multi-spectral MR images using linear discriminant analysis," Comput. Med. Imaging Graphics 34(4), 251-268 (2010).

52. N. V. Reshetov et al., "Principle component analysis and linear discriminant analysis of multi-spectral autofluorescence imaging data for differentiating basal cell carcinoma and healthy skin," Proc. SPIE 9976, 99760B (2016).

53. A. Agresti, An Introduction to Categorical Data Analysis: Second Edition, Wiley, Hoboken, New York (2006).

54. H. H. Hensley et al., "Combined in vivo molecular and anatomic imaging for detection of ovarian carcinoma-associated protease activity and integrin expression in mice," Neoplasia 14(6), 451-462 (2012).

55. Y. Zhang and Q. Chen, "Relationship between matrix metalloproteinases and the occurrence and development of ovarian cancer," Braz. J. Med. Biol. Res. 50(6), e6104 (2017).

56. Q. R. Tummers et al., "Intraoperative imaging of folate receptor alpha positive ovarian and breast cancer using the tumor specific agent EC17," Oncotarget 7(22), 32144-32155 (2016).

57. P. B. Hoyer et al., "Comparison of reproductive function in female TgMISIIR-TAg transgenic and wildtype C57BL/6 Mice," Сотр. Med. 69, 16-21 (2019).

58. T. W. Sawyer et al., "In vivo multiphoton imaging of an ovarian cancer mouse model," Proc SPIE 10856, 1085605 (2019).
Travis W. Sawyer received his BS degree in optical sciences and engineering from the University of Arizona and his MPhil degree in physics from the University of Cambridge. $\mathrm{He}$ is a $\mathrm{PhD}$ student at the University of Arizona, expected to graduate May 2022. His research focuses on developing multimodal imaging systems for cancer surveillance and image analysis for classification.

Jennifer W. Koevary is an assistant professor in biomedical engineering at the University of Arizona and chief operating officer at Avery Therapeutics. Her research interests are primarily in the areas of imaging for cancer detection and evaluation of therapeutic safety and efficacy. She is also interested in the use of engineered human tissues for drug toxicity screening and development of human tissues/ organoids for clinical trials in a dish. She has provisional patents in these areas.

Photini F. S. Rice holds an associate degree in applied science in medical technology and has American Society for Clinical Pathology certification. She worked 13 years as a medical technologist and technical consultant in a clinical laboratory, including achieving CLIA (Clinical Laboratory Improvement Amendments) certification for the laboratory. Currently, she is a senior research specialist at the University of Arizona with 7 years of experience in cardiovascular research and the past 10 years in cancer imaging.

Denise C. Conolly is an associate professor and director of the Biosample Repository Facility at Fox Chase Cancer Center in Philadelphia, Pennsylvania. The overall goal of her research is to discover ways to improve the treatment of epithelial ovarian cancer. In particular, her laboratory is interested in defining molecular mechanisms that contribute to peritoneal dissemination of ovarian cancer cells to identify targets for therapeutic intervention in patients.

Kathy Q. Cai is an assistant research professor and co-director of Histopathology Facility at Fox Chase Cancer Center, Philadelphia, Pennsylvania, USA. Her research interest focuses on developing and improving histopathology-related technologies to support basic and translational research projects involving cell signaling, molecular carcinogenesis, and animal modeling. Her long-term research interests include understanding the molecular mechanism of ovarian cancer initiation and development.

Jennifer K. Barton is a professor of optical sciences and biomedical engineering and the director of the BIO5 Institute at the University of Arizona. Her research interests are in translational biomedical optics and the prevention and early detection of cancer. In particular, her work focuses on technology and application development of optical coherence tomography and fluorescence spectroscopy, and design/development of miniature endoscope-enabled imaging systems.

Biographies of the other authors are not available. 